\title{
Minimal overlapping patterns in colored permutations
}

\author{
Adrian Duane \\ Department of Mathematics \\ University of California, San Diego \\ La Jolla, CA 92093-0112. USA \\ aduane@math.ucsd.edu
}

\author{
Jeffrey Remmel \\ Department of Mathematics \\ University of California, San Diego \\ La Jolla, CA 92093-0112. USA \\ remmel@math.ucsd.edu
}

Submitted: Feb 21, 2011; Accepted: Oct 23, 2011; Published: Oct 31, 2011

MR Subject Classifications: 05A15, 05E05

\section{Dedicated to Doron Zeilberger on the occassion of his sixtieth birthday}

\begin{abstract}
A pattern $P$ of length $j$ has the minimal overlapping property if two consecutive occurrences of the pattern can overlap in at most one place, namely, at the end of the first consecutive occurrence of the pattern and at the start of the second consecutive occurrence of the pattern. For patterns $P$ which have the minimal overlapping property, we derive a general formula for the generating function for the number of consecutive occurrences of $P$ in words, permutations and $k$-colored permutations in terms of the number of maximum packings of $P$ which are patterns of minimal length which has $n$ consecutive occurrences of the pattern $P$. Our results have as special cases several results which have appeared in the literature. Another consequence of our results is to prove a conjecture of Elizalde that two permutations $\alpha$ and $\beta$ of size $j$ which have the minimal overlapping property are strongly $c$-Wilf equivalent if $\alpha$ and $\beta$ have the same first and last elements.
\end{abstract}

\section{Introduction}

For any alphabet $A$, we let $A^{*}$ denote the set of all words over $A$ and we let $\epsilon$ denote the empty word. Given any word $w=w_{1} \ldots w_{n} \in\{0, \ldots, k-1\}^{*}$, we let $\sum w=w_{1}+\cdots+w_{n}$, $|w|=n, z(w)=\prod_{i=1}^{n} z_{w_{i}}$, and $\operatorname{red}(w)$ be the word that results by replacing the $i$-th smallest integer that appears in $w$ by $i-1$. For example, red $(13443551)=01221330$. Given any word $u \in\{0, \ldots, k-1\}^{j}$ such that $\operatorname{red}(u)=u$, we say that a word $w=w_{1} \ldots w_{n} \in$ $\{0, \ldots, k-1\}^{n}$ has a $u$-match starting at position $i$ if $\operatorname{red}\left(w_{i} w_{i+1} \ldots w_{i+j-1}\right)=u$. If $u \in\{0, \ldots, k-1\}^{j}$, then we say that $w=w_{1} \ldots w_{n} \in\{0, \ldots, k-1\}^{n}$ has an exact $u$ match starting at position $i$ if $w_{i} w_{i+1} \ldots w_{i+j-1}=u$. Let $u$-mch $(w)$ denote the number of $u$-matches in the word $w$ and $E u$-mch $(w)$ denote the number of exact $u$-matches in $w$. For example, if $u=010$ and $w=14121010330$, then $w$ has $u$-matches starting at positions 
1,3 , and 5 and has an exact $u$-match starting at position 5 so that $u$-mch $(w)=3$ and $E u-\operatorname{mch}(w)=1$.

Let $S_{n}$ denote the symmetric group. Given any sequence $\sigma=\sigma_{1} \cdots \sigma_{n}$ of distinct integers, we let $\operatorname{pred}(\sigma)$ be the permutation that results by replacing the $i$-th smallest integer that appears in the sequence $\sigma$ by $i$. For example, if $\sigma=2754$, then $\operatorname{pred}(\sigma)=1432$. Given a permutation $\tau=\tau_{1} \ldots \tau_{j}$ in the symmetric group $S_{j}$, we say that a permutation $\sigma=\sigma_{1} \ldots \sigma_{n} \in S_{n}$ has a $\tau$-match at starting at position $i$ if $\operatorname{pred}\left(\sigma_{i} \ldots \sigma_{i+j-1}\right)=\tau$. Let $\tau$-mch $(\sigma)$ be the number of $\tau$-matches in the permutation $\sigma$. We let

$$
\begin{aligned}
{[n]_{p, q} } & =p^{n-1}+p^{n-2} q+\cdots+p q^{n-2}+q^{n-1}=\frac{p^{n}-q^{n}}{p-q} \\
{[n]_{p, q} ! } & =[1]_{q}[2]_{q} \cdots[n]_{q}, \text { and } \\
{\left[\begin{array}{l}
n \\
k
\end{array}\right]_{p, q} } & =\frac{[n]_{p, q} !}{[k]_{p, q} ![n-k]_{p, q} !}
\end{aligned}
$$

denote the usual $p, q$-analogues of $n, n$ !, and $\left(\begin{array}{l}n \\ k\end{array}\right)$. We shall use the standard conventions that $[0]_{p, q}=0$ and $[0]_{p, q} !=1$. Setting $p=1$ in $[n]_{p, q},[n]_{p, q} !$, and $\left[\begin{array}{l}n \\ k\end{array}\right]_{p, q}$ yields $[n]_{q},[n]_{q} !$, and $\left[\begin{array}{l}n \\ k\end{array}\right]_{q}$, respectively. For any permutation $\sigma=\sigma_{1} \ldots \sigma_{n} \in S_{n}$, we let $\operatorname{inv}(\sigma)$ equal the number of $1 \leq i<j \leq n$ such that $\sigma_{i}>\sigma_{j}$ and $\operatorname{coinv}(\sigma)$ equal the number of $1 \leq i<j \leq n$ such that $\sigma_{i}<\sigma_{j}$.

A $k$-colored permutation of size $n$ is a pair $(\sigma, u) \in S_{n} \times\{0,1, \ldots, k-1\}^{n}$ and can be thought of as an element in the wreath product $C_{k} 2 S_{n}$ of the cyclic group $C_{k}$ and the symmetric group $S_{n}$. Thus we will let $C_{k} 2 S_{n}$ denote the set of all $k$-colored permutations of size $n$. Given a pair $(\tau, u) \in C_{k} \zeta S_{j}$ such that $\operatorname{red}(u)=u$, we say that a $k$-colored permutation $(\sigma, w) \in C_{k} 2 S_{n}$ has $(\tau, u)$-match starting at position $i$ if $\operatorname{pred}\left(\sigma_{i} \cdots \sigma_{i+j-1}\right)=$ $\tau$ and $\operatorname{red}\left(w_{i} w_{i+1} \ldots w_{i+j-1}\right)=u$. If $(\tau, u) \in C_{k} \imath S_{j}$, we say that a $k$-colored permutation $(\sigma, w) \in C_{k} \imath S_{n}$ has an exact $(\tau, u)$-match starting at position $i$ if $\operatorname{pred}\left(\sigma_{i} \cdots \sigma_{i+j-1}\right)=\tau$ and $w_{i} w_{i+1} \ldots w_{i+j-1}=u$. Let $(\tau, u)-\operatorname{mch}((\sigma, w))$ denote the number of $(\tau, u)$-matches in $(\sigma, w)$ and $(\tau, E u)-\operatorname{mch}((\sigma, w))$ denote the number of exact $(\tau, u)$-matches in $(\sigma, w)$. For example, if $\sigma=132$ and $u=010$ and $(\sigma, w)=(25316498,14121010)$, then $(\sigma, w)$ has $(\tau, u)$-matches starting at positions 1 and 6 and has an exact $(\tau, u)$-match starting at position 6 so that $(\tau, u)-\operatorname{mch}((\sigma, w))=2$ and $(\tau, E u)-\operatorname{mch}((\sigma, w))=1$.

There are a number of papers on exact $(\tau, u)$-pattern matching and exact $(\tau, u)$-pattern avoidance in $C_{k} 2 S_{n}$, see $[7,16,17,18]$. Our notions of $(\tau, u)$-pattern matching was first introduced in [13] where the authors studied $(\tau, u)$-matches for patterns of length 2 .

Let $u$ be a word in $\{0,1, \ldots, k-1\}^{j}$. If $\operatorname{red}(u)=u$, then we say that $u$ has the $k$ minimal overlapping property if the smallest $i$ such that there exists a $w \in\{0,1, \ldots, k-1\}^{i}$ with $u-\operatorname{mch}(w)=2$ is $2 j-1$. This means that in a word $w \in\{0,1, \ldots, k-1\}^{*}$, two $u$ matches in $w$ can share at most one letter which must occur at the end of the first $u$-match and at the start of the second $u$-match. Similarly, we say that $u$ has the $k$-exact match minimal overlapping property if the smallest $i$ such that there exists a $w \in\{0,1, \ldots, k-1\}^{i}$ with $E u-\operatorname{mch}(w)=2$ is $2 j-1$. For example, it is easy to see that 010 has the both the $k$-minimal overlapping property and the $k$-exact match minimal overlapping property for 
all $k \geq 2$. However, $u=0011$ does not have the $k$-minimal overlapping property for $k \geq 3$ since $u$-mch $(001122)=2$. Also $u=0011$ does not have the $k$-exact matching minimal overlapping property since no two exact $u$-matches can have a letter in common. However, $u=01020$ has the $k$-exact match minimal overlapping property for all $k \geq 3$ and the $k$-minimal overlapping property for $k=3$, but it does not have the $k$-minimal overlapping property for $k \geq 4$ since $u$-mch $(0102030)=2$.

We say that a permutation $\tau \in S_{j}$ where $j \geq 3$ has the minimal overlapping property if the smallest $i$ such that there is a permutation $\sigma \in S_{i}$ with $\tau-\operatorname{mch}(\sigma)=2$ is $2 j-1$. Again this means that in any permutation $\sigma=\sigma_{1} \ldots \sigma_{n}$, any two $\tau$-matches in $\sigma$ can share at most one letter which must be at the end of the first $\tau$-match and the start of the second $\tau$-match. For example $\tau=123$ does not have the minimal overlapping property since the $\tau$-mch $(1234)=2$ and the $\tau$-match starting at position 1 and the $\tau$-match starting at position 2 share two letters, namely, 2 and 3 . However, it is easy to see that the permutation $\tau=132$ does have the minimal overlapping property. That is, the fact that there is an ascent starting at position 1 and descent starting at position 2 means that there cannot be two $\tau$-matches in a permutation $\sigma \in S_{n}$ which share 2 or more letters.

Suppose that we are given $u \in\{0,1, \ldots, k-1\}^{j}$ and $\tau \in S_{j}$. If $\operatorname{red}(u)=u$, then we say that $u$ has the $C_{k} 2 S_{n}$-minimal overlapping property if the smallest $i$ such that there exists a $(\sigma, w) \in C_{k}$ ? $S_{i}$ with $(\tau, u)-\operatorname{mch}(w)=2$ is $2 j-1$. This means that in a $k$-colored permutation $(\sigma, w)$, two $(\tau, u)$-matches in $(\sigma, w)$ can share at most one pair of letters which must occur at the end of the first $(\tau, u)$-match and at the start of the second $(\tau, u)$-match. Similarly, we say that $(\tau, u)$ has the $C_{k}$ 2 $S_{n}$-exact match minimal overlapping property if the smallest $i$ such that there exists a $(\sigma, w) \in C_{k} \imath S_{i}$ with $(\tau, E u)-\operatorname{mch}((\sigma, w))=2$ is $2 j-1$. For example, it is easy to see that $(132,010)$ has the both the $C_{k} 2 S_{n}$-minimal overlapping property and the $C_{k}$ 2 $S_{n}$-exact match minimal overlapping property for all $k \geq 2$. However, $(\tau, u)=(2143,0011)$ does not have the $C_{k} 2 S_{n}$-minimal overlapping property for $k \geq 3$ since $(\tau, u)-\operatorname{mch}((214365,001122))=2$. Note $(\tau, u)=(2143,0011)$ does not have the $C_{k}$ 々 $S_{n}$-exact match minimal overlapping property since no two exact $(\tau, u)$-matches can have a pair of letters in common. Also $(\tau, u)=(12345,01020)$ has the $C_{k} \prec S_{n}$-exact match minimal overlapping property for all $k \geq 3$ and the $C_{k}$ 々 $S_{n}$-minimal overlapping property for $k=3$, but it does not have the $C_{k}$ 2 $S_{n}$-minimal overlapping property for $k \geq 4$ since $(\tau, u)-\operatorname{mch}((1234567,0102030))=2$.

The main goal of this paper is to find generating functions for the number of matches of minimal overlapping patterns in words, permutations, and $k$-colored permutations. To this end, suppose that $u \in\{0,1, \ldots, k-1\}^{j}$. If $\operatorname{red}(u)=u$ and $u$ has the $k$-minimal overlapping property, then the shortest words $w \in\{0,1, \ldots, k-1\}^{*}$ such that $u$-mch $(w)=$ $n$ have length $j+(n-1)(j-1)=n(j-1)+1$ so we let $\mathcal{M P}_{u, n(j-1)+1}^{k}$ denote the set of words $w \in\{0,1, \ldots, k-1\}^{n(j-1)+1}$ such that $u-\operatorname{mch}(w)=n$. We will refer to elements of $\mathcal{M} \mathcal{P}_{u, n(j-1)+1}^{k}$ as maximum packings for $u$. We let

$$
\begin{aligned}
& m p_{u, n(j-1)+1}^{k}=\left|\mathcal{M} \mathcal{P}_{u, n(j-1)+1}^{k}\right|, \\
& m p_{u, n(j-1)+1}^{k}(r)=\sum_{w \in \mathcal{M} \mathcal{P}_{u, n(j-1)+1}^{k}} r^{\sum w}, \text { and }
\end{aligned}
$$




$$
m p_{u, n(j-1)+1}^{k}\left(z_{0}, \ldots, z_{k-1}\right)=\sum_{w \in \mathcal{M} \mathcal{P}_{u, n(j-1)+1}^{k}} z(w) .
$$

If $u$ has the $k$-exact match minimal overlapping property, then the shortest words $w \in$ $\{0,1, \ldots, k-1\}^{*}$ such that $E u-\operatorname{mch}(w)=n$ have length $n(j-1)+1$ so we let $\mathcal{E} \mathcal{M} \mathcal{P}_{u, n(j-1)+1}^{k}$ denote the set of words $w \in\{0,1, \ldots, k-1\}^{n(j-1)+1} \operatorname{such}$ that $E u$-mch $(w)=n$. We will refer to elements of $\mathcal{E M}_{\mathcal{M}}^{k}{ }_{u, n(j-1)+1}$ as exact match maximum packings for $u$. We let

$$
\begin{aligned}
& e m p_{u, n(j-1)+1}^{k}=\left|\mathcal{E} \mathcal{M} \mathcal{P}_{u, n(j-1)+1}^{k}\right| \\
& e m p_{u, n(j-1)+1}^{k}(r)=\sum_{w \in \mathcal{E} \mathcal{M} \mathcal{P}_{u, n(j-1)+1}^{k}} r^{\sum w} \text {, and } \\
& e m p_{u, n(j-1)+1}^{k}\left(z_{0}, \ldots, z_{k-1}\right)=\sum_{w \in \mathcal{E} \mathcal{M} \mathcal{P}_{u, n(j-1)+1}^{k}} z(w) .
\end{aligned}
$$

For example, suppose that $u=010$ and $k \geq 2$. Then the only $w \in\{0,1, \ldots, k-1\}^{2 n+1}$ such that $E u-\operatorname{mch}(w)=n$ is $0(10)^{n}$ so that $e m p_{010,2 n+1}^{k}=1, e m p_{010,2 n+1}(r)=r^{n}$, and $e m p_{010, n(j-1)+1}^{k}\left(z_{0}, \ldots, z_{k-1}\right)=z_{0}^{n+1} z_{1}^{n}$ for all $n \geq 1$ and $k \geq 2$. However, if we are just considering $u$-matches instead of exact $u$-matches in $\{0,1, \ldots, k-1\}^{2 n+1}$, then the only words $w \in\{0,1, \ldots, k-1\}^{2 n+1}$ such that $u-\operatorname{mch}(w)=n$ are of the form $s i_{1} s i_{2} j \ldots s i_{n} s$ where $s \in\{0,1, \ldots, k-2\}$ and $i_{1}, \ldots, i_{n} \in\{s+1, \ldots, k-1\}$. Thus

$$
\begin{aligned}
& m p_{010,2 n+1}^{k}=\sum_{s=0}^{k-2}(k-1-s)^{n}=\sum_{i=1}^{k-1} i^{n}, \\
& m p_{010,2 n+1}^{k}(r)=\sum_{s=0}^{k-2} r^{(n+1) s}\left(r^{s+1}[k-1-s]_{r}\right)^{n}=\sum_{i=1}^{k-1} r^{2 n(k-i)-n-1}[i]_{r}^{n}, \text { and } \\
& m p_{010,2 n+1}^{k}\left(z_{0}, \ldots, z_{k-1}\right)=\sum_{s=0}^{k-2} z_{s}^{n+1}\left(\sum_{t=s+1}^{k-1} z_{t}\right)^{n} .
\end{aligned}
$$

If $\tau \in S_{j}$ has the minimal overlapping property, then again the shortest permutations $\sigma$ such that $\tau-\operatorname{mch}(\sigma)=n$ have length $n(j-1)+1$. Thus we let $\mathcal{M} \mathcal{P}_{\tau, n(j-1)+1}$ equal the set of permutations $\sigma \in S_{n(j-1)+1}$ such that $\tau-\operatorname{mch}(\sigma)=n$. Again we refer to the permutations in $\mathcal{M P}_{n, n(j-1)+1}$ as maximum packings for $\tau$. Then we let $m p_{\tau, n(j-1)+1}=\left|\mathcal{M} \mathcal{P}_{\tau, n(j-1)+1}\right|$ and

$$
m p_{\tau, n(j-1)+1}(p, q)=\sum_{\sigma \in \mathcal{M} \mathcal{P}_{\tau, n(j-1)+1}} q^{\operatorname{inv}(\sigma)} p^{\operatorname{coinv}(\sigma)} .
$$

In general, it is a difficult problem to compute $m p_{\tau, n(j-1)+1}$ or $m p_{\tau, n(j-1)+1}(p, q)$, but we can compute these in the case that $\tau$ starts either ends or starts with 1 or ends or starts with $j$. For example, we shall prove the following theorem.

Theorem 1. Suppose that $\tau=\tau_{1} \ldots \tau_{j}$ where $\tau_{1}=1$ and $\tau_{j}=s$, then 


$$
\begin{aligned}
& m p_{\tau,(n+1)(j-1)+1}(p, q)= \\
& p^{\operatorname{coinv}(\tau)} q^{i n v(\tau)} p^{(s-1) n(j-1)}\left[\begin{array}{c}
(n+1)(j-1)+1-s \\
j-s
\end{array}\right]_{p, q} m p_{\tau, n(j-1)+1}(p, q)
\end{aligned}
$$

so that

$$
m p_{\tau,(n+1)(j-1)+1}(p, q)=\left(p^{\operatorname{coinv}(\tau)} q^{i n v(\tau)}\right)^{n+1} p^{(s-1)(j-1)\left(\begin{array}{c}
n+1 \\
2
\end{array}\right)} \prod_{i=1}^{n+1}\left[\begin{array}{c}
i(j-1)+1-s \\
j-s
\end{array}\right]_{p, q} .
$$

Note that if $\tau=\tau_{1} \ldots \tau_{j} \in S_{j}$ has the minimal overlapping property, then the reverse of $\tau, \tau^{r}=\tau_{j} \ldots \tau_{1}$, and the complement of $\tau, \tau^{c}=\left(j+1-\tau_{1}\right) \ldots\left(j+1-\tau_{j}\right)$, also have the minimal overlapping property. Thus one can use Theorem 1 to compute $m p_{\tau,(n+1)(j-1)+1}(p, q)$ in the case where $\tau$ either ends or starts with $j$ or ends with 1 .

Now suppose that $u \in\{0,1, \ldots, k-1\}^{j}$ and $\tau \in S_{j}$. If $\operatorname{red}(u)=u$ and $(\tau, u)$ has the $C_{k} 2 S_{n}$-minimal overlapping property, then the shortest $k$-colored permutations $(\sigma, w)$ such that $(\tau, u)-\operatorname{mch}((\sigma, w))=n$ have length $n(j-1)+1$. Thus we let $\mathcal{M} \mathcal{P}_{(\tau, u), n(j-1)+1}^{k}$ equal the set of $k$-colored permutations $(\sigma, w) \in C_{k} 2 S_{n(j-1)+1} \operatorname{such}$ that $(\tau, u)-\operatorname{mch}((\sigma, w))=n$. We refer to the $k$-colored permutations in $\mathcal{M P}_{(\tau, u), n(j-1)+1}^{k}$ as maximum packings for $(\tau, u)$. We let

$$
\begin{aligned}
& m p_{(\tau, u), n(j-1)+1}^{k}=\left|\mathcal{M} \mathcal{P}_{(\tau, u), n(j-1)+1}^{k}\right|, \\
& m p_{(\tau, u), n(j-1)+1}^{k}(p, q, r)=\sum_{(\sigma, w) \in \mathcal{M} \mathcal{P}_{(\tau, u), n(j-1)+1}^{k}} p^{\operatorname{coinv}(\sigma)} q^{\operatorname{inv}(\sigma)} r^{\sum w} \text {, and } \\
& m p_{(\tau, u), n(j-1)+1}^{k}\left(p, q, z_{0}, \ldots, z_{k-1}\right)=\sum_{(\sigma, w) \in \mathcal{M} \mathcal{P}_{(\tau, u), n(j-1)+1}^{k}} p^{\operatorname{coinv}(\sigma)} q^{\operatorname{inv}(\sigma)} z(w) .
\end{aligned}
$$

Similarly, we let $\mathcal{E M P P}_{(\tau, u), n(j-1)+1}^{k}$ equal the set of $(\sigma, w) \in C_{k} 2 S_{n(j-1)+1}$ such that

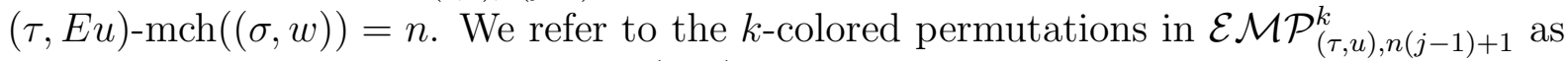
exact match maximum packings for $(\tau, u)$. We let

$$
\begin{aligned}
& e m p_{(\tau, u), n(j-1)+1}^{k}=\left|\mathcal{E} \mathcal{M} \mathcal{P}_{(\tau, u), n(j-1)+1}^{k}\right|, \\
& e m p_{(\tau, u), n(j-1)+1}^{k}(p, q, r)=\sum_{(\sigma, w) \in \mathcal{E} \mathcal{M} \mathcal{P}_{(\tau, u), n(j-1)+1}^{k}} p^{\operatorname{coinv}(\sigma)} q^{\operatorname{inv}(\sigma)} r^{\sum w} \text {, and } \\
& e m p_{(\tau, u), n(j-1)+1}^{k}\left(p, q, z_{0}, \ldots, z_{k-1}\right)=\sum_{(\sigma, w) \in \mathcal{E} \mathcal{M} \mathcal{P}_{(\tau, u), n(j-1)+1}^{k}} p^{\operatorname{coinv}(\sigma)} q^{\operatorname{inv}(\sigma)} z(w) .
\end{aligned}
$$

Then the main goal of this paper is to prove the following. Let $u \in\{0,1, \ldots, k-1\}^{j}$ and $\tau \in S_{j}$ where $j \geq 3$.

(I) If $\operatorname{red}(u)=u$ and $u$ has the $k$-minimal overlapping property, then

$$
\sum_{n \geq 0} t^{n} \sum_{w \in\{0,1, \ldots, k-1\}^{n}} x^{u-\operatorname{mch}(w)} z(w)=
$$




$$
\frac{1}{1-\left(\left(z_{0}+\cdots+z_{k-1}\right) t+\sum_{n \geq 1} t^{n(j-1)+1}(x-1)^{n} m p_{u, n(j-1)+1}^{k}\left(z_{0}, \ldots, z_{k-1}\right)\right)} .
$$

(II) If $u$ has the $k$-exact match minimal overlapping property, then

$$
\begin{aligned}
\sum_{n \geq 0} t^{n} & \sum_{w \in\{0,1, \ldots, k-1\}^{n}} x^{E u-\operatorname{mch}(w)} z(w)= \\
& \frac{1}{1-\left(\left(z_{0}+\cdots+z_{k-1}\right) t+\sum_{n \geq 1} t^{n(j-1)+1}(x-1)^{n} e m p_{u, n(j-1)+1}^{k}\left(z_{0}, \ldots, z_{k-1}\right)\right)} .
\end{aligned}
$$

(III) If $\operatorname{red}(u)=u$ and $(\tau, u)$ has the $C_{k} \imath S_{n}$-minimal overlapping property, then

$$
\begin{aligned}
& \sum_{n \geq 0} \frac{t^{n}}{n !} \sum_{(\sigma, w) \in C_{k} S_{n}} x^{(\tau, u)-\operatorname{mch}((\sigma, u))} p^{\operatorname{coinv}(\sigma)} q^{\operatorname{inv}(\sigma)} z(w)= \\
& 1-\left(\left(z_{0}+\cdots+z_{k-1}\right) t+\sum_{n \geq 1} \frac{t^{n(j-1)+1}}{[n(j-1)+1]_{p, q} !}(x-1)^{n} m p_{(\tau, u), n(j-1)+1}^{k}\left(p, q, z_{0}, \ldots, z_{k-1}\right)\right)
\end{aligned}
$$

(IV) If $(\tau, u) \in C_{k} \prec S_{j}$ has the $C_{k} \prec S_{n}$-exact match minimal overlapping property, then

$$
\begin{aligned}
& \sum_{n \geq 0} \frac{t^{n}}{n !} \sum_{(\sigma, w) \in C_{k} l S_{n}} x^{(\tau, E u)-\operatorname{mch}((\sigma, u))} p^{\operatorname{coinv}(\sigma)} q^{\operatorname{inv}(\sigma)} z(w)= \\
& \frac{1}{1-\left(\left(z_{0}+\cdots+z_{k-1}\right) t+\sum_{n \geq 1} \frac{t^{n(j-1)+1}}{n(j-1)+1]_{q} !}(x-1)^{n} e m p_{(\tau, u), n(j-1)+1}^{k}\left(p, q, z_{0}, \ldots, z_{k-1}\right)\right)} .
\end{aligned}
$$

The special case of (3) or (4) when $k=1$ proves the following result.

(V) If $\tau \in S_{j}$ has the minimal overlapping property, then

$$
\begin{aligned}
\sum_{n \geq 0} \frac{t^{n}}{n !} \sum_{\sigma \in S_{n}} x^{\tau-\operatorname{mch}(\sigma)} p^{\operatorname{coinv}(\sigma)} q^{\operatorname{inv}(\sigma)}= & \frac{1}{1-\left(t+\sum_{n \geq 1} \frac{t^{n(j-1)+1}}{[n(j-1)+1]_{p, q} !}(x-1)^{n} m p_{\tau, n(j-1)+1}(p, q)\right)} .
\end{aligned}
$$

We shall prove all of our generating functions by applying an appropriate ring homomorphism, defined on the ring $\Lambda$ of symmetric functions over infinitely many variables $x_{1}, x_{2}, \ldots$, to a simple symmetric function identity. There has been a long line of research, [2], [3], [1] [14], [15], [19], [20], [21], [23], [25], [22], which shows that a large number of generating functions for permutation statistics can be obtained by applying homomorphisms defined on $\Lambda$ to simple symmetric function identities. For example, the $n$-th elementary symmetric function, $e_{n}$, and the $n$-th homogeneous symmetric function, $h_{n}$, are defined by the generating functions

$$
E(t)=\sum_{n \geq 0} e_{n} t^{n}=\prod_{i}\left(1+x_{i} t\right)
$$


and

$$
H(t)=\sum_{n \geq 0} h_{n} t^{n}=\prod_{i} \frac{1}{1-x_{i} t} .
$$

Thus

$$
H(t)=1 / E(-t)
$$

It is well known that $\left\{e_{0}, e_{1}, \ldots\right\}$ is an algebraically independent set of generators for $\Lambda$ and hence we can define a ring homomorphism $\xi: \Lambda \rightarrow R$ where $R$ is a ring by simply specifying $\xi\left(e_{n}\right)$ for all $n \geq 0$. We shall prove (1)-(5) by applying appropriate ring homomorphisms to (9).

We shall show that several theorems that have been proved in the literature are special cases of (I)-(V). For example, let

$$
A_{\tau}(t)=\sum_{n \geq 0} \frac{t^{n}}{n !}\left|\left\{\sigma \in S_{n}: \tau-\operatorname{mch}(\sigma)=0\right\}\right|
$$

and

$$
P_{\tau}(t, x)=\sum_{n \geq 0} \frac{t^{n}}{n !} \sum_{\sigma \in S_{n}} x^{\tau-\operatorname{mch}(\sigma)} .
$$

Elizalde and Noy [8] proved a number of results about $A_{\tau}(t)$ and $P_{\tau}(x, t)$. For example, they showed that

$$
\begin{aligned}
P_{132}(t, x) & =\frac{1}{1-\int_{0}^{t} e^{\frac{(x-1) z^{2}}{2}} d z}, \text { and } \\
P_{1342}(t, x) & =\frac{1}{1-\int_{0}^{t} e^{\frac{(x-1) z^{3}}{6}} d z} .
\end{aligned}
$$

Later Kitaev [11] used an inclusion exclusion argument to prove the following result.

Theorem 2. Let $\tau=12 \cdots a \sigma(a+1)$, where $\sigma$ is a permutation of $\{a+2, a+3, \ldots, k+1\}$, then

$$
A_{\tau}(t)=\frac{1}{1-t+\sum_{i \geq 1} \frac{(-1)^{i+1} t^{k i+1}}{(k i+1) !} \prod_{j=2}^{i}\left(\begin{array}{c}
j k-a \\
k-a
\end{array}\right)} .
$$

Permutations of the form 132, 1342, and $\tau=12 \cdots a \sigma(a+1)$ have the minimal overlapping property so that $p, q$-analogues of the results of Elizalde and Noy and Kitaev's result are special cases of our main results.

We shall see that our results have many applications to the consecutive Wilf equivalence problem. That is, given $\alpha, \beta \in S_{n}$, we say that $\alpha$ is c-Wilf equivalent to $\beta$ if $A_{\alpha}(t)=A_{\beta}(t)$. Given permutations $\alpha, \beta \in S_{n}$, we say that $\alpha$ is strongly c-Wilf equivalent to $\beta$ if $P_{\alpha}(t, x)=P_{\beta}(t, x)$. It is easy to see that $\mathrm{c}$-Wilf equivalences classes and strong c-Wilf equivalence classes are closed under complement and reverse.

Elizalde [7] conjectured that if $\alpha=\alpha_{1} \ldots \alpha_{j}$ and $\beta=\beta_{1} \ldots \beta_{j}$ are permutations in $S_{j}$ which have the minimal overlapping property and $\alpha_{1}=\beta_{1}$ and $\alpha_{j}=\beta_{j}$, then $\alpha$ and 
$\beta$ are strongly c-Wilf equivalent. Note that (5) tells us that if $\alpha$ and $\beta$ are elements of $S_{j}$ which have the minimal overlapping property and $m p_{\alpha, s(j-1)+1}=m p_{\beta, s(j-1)+1}$ for all $s \geq 1$, then $P_{\alpha}(x, t)=P_{\beta}(x, t)$ and, hence, $\alpha$ and $\beta$ are strongly $\mathrm{c}-W i l f$ equivalent. Thus to prove Elizalde's conjecture, we need only show that if $\alpha=\alpha_{1} \ldots \alpha_{j}$ and $\beta=\beta_{1} \ldots \beta_{j}$ are elements of $S_{j}$ which have minimal overlapping property and $\alpha_{1}=\beta_{1}$ and $\alpha_{j}=\beta_{j}$, then $m p_{\alpha, s(j-1)+1}=m p_{\beta, s(j-1)+1}$ for all $s \geq 1$. We shall prove this fact in Section 3 . We note that Elizalde's conjecture has been proved independently by Vladimir Dotsenko and Anton Khoroshkin [4] by a different method.

Our results will also allow us to find generating functions for the distribution for the number of non-overlapping matches of a pattern in permutations, words, and colored permutations. That is, if $u \in\{0,1, \ldots, k-1\}^{j}$ is such that $\operatorname{red}(u)=u$ and $w \in\{0,1, \ldots, k-1\}^{n}$, then we let $u$-nlap $(w)$ denote the maximum number of nonoverlapping $u$-matches in $w$ where we say that two $u$-matches in $w$ overlap that they have at least one position in common. Similarly, we let $E u$-nlap $(w)$ denote the maximum number of non-overlapping exact $u$-matches in $w$. If $\tau \in S_{j}$ and $\sigma \in S_{n}$, let $\tau$-nlap $(\sigma)$ denote the maximum number of non-overlapping $\tau$ matches in $\sigma$. If $(\tau, u) \in C_{k} \zeta S_{j}$ is such that $\operatorname{red}(u)=u$ and $(\sigma, w) \in C_{k} 2 S_{n}$, then we let $(\tau, u)-\operatorname{nlap}((\sigma, w))$ denote the maximum number of non-overlapping $(\tau, u)$-matches in $(\sigma, w)$. Similarly, if $(\tau, u) \in C_{k} \imath S_{j}$, then we let $(\tau, E u)-\operatorname{nlap}((\sigma, w))$ denote the maximum number of non-overlapping exact $(\tau, u)$-matches in $(\sigma, w)$.

Kitaev $[10,11]$ showed that if one can compute $A_{\tau}(t)$, then one can automatically compute the exponential generating function for the distribution of $\tau$-nlap $(\sigma)$. That is, Kitaev [10, 11] proved that

Theorem 3.

$$
\sum_{n \geq 0} \frac{t^{n}}{n !} \sum_{\sigma \in S_{n}} x^{\tau-\operatorname{nlap}(\tau) \sigma}=\frac{A_{\tau}(t)}{(1-x)+x(1-t) A_{\tau}(t)}
$$

where $A_{\tau}(t)=\sum_{n \geq 0} \frac{t^{n}}{n !}\left|\left\{\sigma \in S_{n}: \tau-\operatorname{mch}(\tau) \sigma=0\right\}\right|$.

Mendes and Remmel [19] proved a $q$-analogue of Theorem 3. That is, they proved that

$$
\sum_{n=0}^{\infty} \frac{t^{n}}{[n]_{q} !} \sum_{\sigma \in S_{n}} x^{\tau-\operatorname{nlap}(\sigma)} q^{i n v(\sigma)}=\frac{A_{\tau}(q, t)}{(1-x)+x(1-t) A_{\tau}(q, t)}
$$

where $A_{\tau}(q, t)=\sum_{n \geq 0} \frac{t^{n}}{[n]_{q} !} \sum_{\sigma \in S_{n}, \tau-\operatorname{mch}(\sigma)=0} q^{\operatorname{inv}(\sigma)}$. Kitaev and Mansour [12] proved an analogue of Theorem 3 for words. That is, they proved that if $u \in\{0,1, \ldots, k-1\}^{*}$, then

$$
\sum_{n \geq 0} t^{n} \sum_{w \in\{0,1, \ldots, k-1\}^{n}} x^{E u-\operatorname{nlap}(w)}=\frac{A_{u}(t)}{1-x\left((k t-1) A_{u}(t)\right)}
$$

where $A_{u}(t)=\sum_{n \geq 0} t^{n}\left|\left\{w \in\{0,1, \ldots, k-1\}^{n}: \operatorname{Eu-mch}(w)=0\right\}\right|$. One can easily modify Kitaev and Mansour's proof to prove the following refinement of their results. That is, if

$$
A_{u}\left(t, z_{0}, \ldots, z_{k-1}\right)=\sum_{n \geq 0} t^{n} \sum_{w \in\{0,1, \ldots, k-1\}^{n}, u-\operatorname{mch}(w)=0} z(w),
$$




$$
\begin{aligned}
E A_{u}\left(t, z_{0}, \ldots, z_{k-1}\right) & =\sum_{n \geq 0} t^{n} \sum_{w \in\{0,1, \ldots, k-1\}^{n}, E u-\operatorname{mch}(w)=0} z(w), \\
N_{u}\left(t, x, z_{0}, \ldots, z_{k-1}\right) & =\sum_{n \geq 0} t^{n} \sum_{w \in\{0,1, \ldots, k-1\}^{n}} x^{u-\operatorname{nlap}((\sigma, w))} z(w), \text { and } \\
E N_{u}\left(t, x, z_{0}, \ldots, z_{k-1}\right) & =\sum_{n \geq 0} t^{n} \sum_{w \in\{0,1, \ldots, k-1\}^{n}}^{E u-\operatorname{nlap}((\sigma, w))} z(w),
\end{aligned}
$$

then

$$
\begin{aligned}
N_{u}\left(t, x, z_{0}, \ldots, z_{k-1}\right) & =\frac{A_{u}\left(t, z_{0}, \ldots, z_{k-1}\right)}{1-x\left(1+\left(\left(z_{0}+\cdots z_{k-1}\right) t-1\right) A_{u}\left(t, z_{0}, \ldots, z_{k-1}\right)\right)} \text { and } \\
E N_{u}\left(t, x, z_{0}, \ldots, z_{k-1}\right) & =\frac{E A_{u}\left(t, z_{0}, \ldots, z_{k-1}\right)}{1-x\left(1+\left(\left(z_{0}+\cdots z_{k-1}\right) t-1\right) E A_{u}\left(t, z_{0}, \ldots, z_{k-1}\right)\right)} .
\end{aligned}
$$

Kitaev, Niedermaier, Remmel and Reihl [13] proved an analogue of Kitaev's theorem for $k$-colored permutations. That is, let

$$
N_{(\tau, u)}(x, r, q, t)=\sum_{n \geq 0} \frac{t^{n}}{[n]_{q} !} \sum_{(\sigma, w) \in C_{k} 2 S_{n}} q^{\operatorname{inv}(\sigma)} r^{\sum w} x^{(\tau, u)-\operatorname{nlap}((\sigma, w))},
$$

and

$$
A_{(\tau, u)}(r, q, t)=\sum_{n \geq 0} \frac{t^{n}}{[n]_{q} !} \sum_{(\sigma, w) \in C_{k}\left(S_{n},(\tau, u)-\operatorname{mch}((\sigma, w))=0\right.} q^{\operatorname{inv}(\sigma)} r^{\sum w} .
$$

Then they showed that

$$
N_{(\tau, u)}(x, r, q, t)=\frac{A_{(\tau, u)}(r, q, t)}{1-x\left(1+\left([k]_{r} t-1\right) A_{(\tau, u)}(r, q, t)\right)} .
$$

One can easily modify the proofs in [13] to obtain the following refinements of their results:

$$
\begin{aligned}
N_{(\tau, u)}\left(t, x, p, q, z_{0}, \ldots, z_{k-1}\right) & = \\
& \frac{A_{(\tau, u)}\left(t, p, q, z_{0}, \ldots, z_{k-1}\right)}{1-x\left(1+\left(\left(z_{0}+\cdots z_{k-1}\right) t-1\right) A_{(\tau, u)}\left(t, p, q, z_{0}, \ldots, z_{k-1}\right)\right)}
\end{aligned}
$$

and

$$
\begin{aligned}
& E N_{(\tau, u)}\left(t, x, p, q, z_{0}, \ldots, z_{k-1}\right)= \\
& \frac{A_{(\tau, u)}\left(t, p, q, z_{0}, \ldots, z_{k-1}\right)}{1-x\left(1+\left(\left(z_{0}+\cdots z_{k-1}\right) t-1\right) E A_{(\tau, u)}\left(t, p, q, z_{0}, \ldots, z_{k-1}\right)\right)}
\end{aligned}
$$

where

$$
A_{(\tau, u)}\left(t, p, q, z_{0}, \ldots, z_{k-1}\right)=\sum_{n \geq 0} \frac{t^{n}}{[n]_{p, q} !} \sum_{(\sigma, w) \in C_{k} l S_{n},(\tau, u)-\operatorname{mch}((\sigma, w))=0} p^{\operatorname{coinv}(\sigma)} q^{\operatorname{inv}(\sigma)} z(w)
$$




$$
\begin{aligned}
E A_{(\tau, u)}\left(t, p, q, z_{0}, \ldots, z_{k-1}\right) & =\sum_{n \geq 0} \frac{t^{n}}{[n]_{p, q} !} \sum_{(\sigma, w) \in C_{k} l S_{n},(\tau, E u)-\operatorname{mch}((\sigma, w))=0} p^{\operatorname{coinv}(\sigma)} q^{\operatorname{inv}(\sigma)} z(w), \\
N_{(\tau, u)}\left(t, x, p, q, z_{0}, \ldots, z_{k-1}\right) & =\sum_{n \geq 0} \frac{t^{n}}{[n]_{p, q} !} \sum_{(\sigma, w) \in C_{k} l S_{n}} p^{\operatorname{coinv}(\sigma)} q^{\operatorname{inv}(\sigma)} x^{(\tau, u)-\operatorname{nlap}((\sigma, w))} z(w),
\end{aligned}
$$

and

$$
E N_{(\tau, u)}\left(t, p, q, z_{0}, \ldots, z_{k-1}\right)=\sum_{n \geq 0} \frac{t^{n}}{[n]_{p, q} !} \sum_{(\sigma, w) \in C_{k} l S_{n}} p^{\operatorname{coinv}(\sigma)} q^{\operatorname{inv}(\sigma)} x^{(\tau, E u)-\operatorname{nlap}((\sigma, w))} z(w) .
$$

The outline of this paper is as follows. In section 2, we shall give the necessary background on symmetric functions and brick tabloids that we shall need for our results. In section 3, we shall prove results (I)-(V) listed above. We shall also show that we can obtain the generating functions for the number of consecutive occurrences in words and $k$-colored permutations of patterns which do not allow any overlaps as special cases of our proofs of $(\mathrm{I})-(\mathrm{V})$. In section 4 , we shall compute the number of maximum packings for various words, permutations, and $k$-colored permutations and show how Elizalde's conjecture follows from our results. Finally, in section 5, we shall briefly discuss some extensions of our results.

\section{Symmetric Functions}

In this section we give the necessary background on symmetric functions needed for our proofs of the generating functions (I)-(IV).

Let $\Lambda$ denote the ring of symmetric functions over infinitely many variables $x_{1}, x_{2}, \ldots$ with coefficients in the field of complex numbers $\mathbb{C}$.

Let $\lambda=\left(\lambda_{1}, \ldots, \lambda_{\ell}\right)$ be an integer partition, that is, $\lambda$ is a finite sequence of weakly increasing nonnegative integers. Let $\ell(\lambda)$ denote the number of nonzero integers in $\lambda$. If the sum of these integers is $n$, we say that $\lambda$ is a partition of $n$ and write $\lambda \vdash n$. For any partition $\lambda=\left(\lambda_{1}, \ldots, \lambda_{\ell}\right)$, let $e_{\lambda}=e_{\lambda_{1}} \cdots e_{\lambda_{\ell}}$. The well-known fundamental theorem of symmetric functions says that $\left\{e_{\lambda}: \lambda\right.$ is a partition $\}$ is a basis for $\Lambda$ or, equivalently, that $\left\{e_{0}, e_{1}, \ldots\right\}$ is an algebraically independent set of generators for $\Lambda$. Similarly, if we define $h_{\lambda}=h_{\lambda_{1}} \cdots h_{\lambda_{\ell}}$, then $\left\{h_{\lambda}: \lambda\right.$ is a partition $\}$ is also a basis for $\Lambda$. Since $\left\{e_{0}, e_{1}, \ldots\right\}$ is an algebraically independent set of generators for $\Lambda$, we can specify a ring homomorphism $\theta$ on $\Lambda$ by simply defining $\theta\left(e_{n}\right)$ for all $n \geq 0$.

A brick tabloid of shape $(n)$ and type $\lambda=\left(\lambda_{1}, \ldots, \lambda_{k}\right)$ is a filling of a row of $n$ squares of cells with bricks of lengths $\lambda_{1}, \ldots, \lambda_{k}$ such that bricks to not overlap. One brick tabloid of shape (12) and type $(1,1,2,3,5)$ is displayed below.

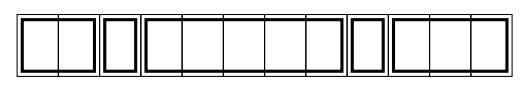

Figure 1: A brick tabloid of shape (12) and type $(1,1,2,3,5)$. 
Let $\mathcal{B}_{\lambda, n}$ denote the set of all $\lambda$-brick tabloids of shape $(n)$ and let $B_{\lambda, n}=\left|\mathcal{B}_{\lambda, n}\right|$. Eğecioğlu and Remmel proved in [5] that

$$
h_{n}=\sum_{\lambda \vdash n}(-1)^{n-\ell(\lambda)} B_{\lambda, n} e_{\lambda} .
$$

We end this section with a lemma from [22] that will be needed in later sections. Fix a brick tabloid $T=\left(b_{1}, \ldots, b_{\ell(\mu)}\right) \in \mathcal{B}_{\mu, n}$. Let $I F(T)$ denote the set of all fillings of the cells of $T=\left(b_{1}, \ldots, b_{\ell(\mu)}\right)$ with the numbers $1, \ldots, n$ so that the numbers increase within each brick reading from left to right. We then think of each such filling as a permutation of $S_{n}$ by reading the numbers from left to right. For example, Figure 2 pictures an element of IF $(3,6,3)$ whose corresponding permutation is 461215781011239 .

$$
\begin{array}{|l|l|l|l|l|l|l|l|l|l|l|l|}
\hline 4 & 6 & 12 & 1 & 5 & 7 & 8 & 10 & 11 & 2 & 3 & 9 \\
\hline
\end{array}
$$

Figure 2: An element of $\operatorname{IF}(3,6,3)$.

Then the following lemma which is proved in [22] gives a combinatorial interpretation to $p^{\sum_{i=1}^{\ell(\mu)}\left(\begin{array}{c}b_{i} \\ 2\end{array}\right)}\left[\begin{array}{c}n \\ b_{1}, \ldots, b_{\ell(\mu)}\end{array}\right]$ p,q

Lemma 4. If $T=\left(b_{1}, \ldots, b_{\ell(\mu)}\right)$ is a brick tabloid in $\mathcal{B}_{\mu, n}$, then

$$
p^{\sum_{i}\left(\begin{array}{c}
b_{i} \\
2
\end{array}\right)}\left[\begin{array}{c}
n \\
b_{1}, \ldots, b_{\ell(\mu)}
\end{array}\right]_{p, q}=\sum_{\sigma \in I F(T)} q^{i n v(\sigma)} p^{\operatorname{coinv}(\sigma)}
$$

\section{Generating functions for minimal overlapping pat- terns}

The main goal of this section is to prove results (I)-(V) described in the introduction. We shall start with results (I) and (II) since their proofs will illustrate the general method.

Theorem 5. Let $u \in\{0,1, \ldots, k-1\}^{j}$ where $j \geq 3$.

(I) If $u$ has the $k$-minimal overlapping property and $\operatorname{red}(u)=u$, then

$$
\begin{aligned}
& \sum_{n \geq 0} t^{n} \sum_{w \in\{0,1, \ldots, k-1\}^{n}} x^{u-\operatorname{mch}(w)} z(w)= \\
& \frac{1}{1-\left(\left(z_{0}+\cdots+z_{k-1}\right) t+\sum_{n \geq 1} t^{n(j-1)+1}(x-1)^{n} m p_{u, n(j-1)+1}^{k}\left(z_{0}, \ldots, z_{k-1}\right)\right)} .
\end{aligned}
$$

(II) If $u$ has the $k$-exact match minimal overlapping property, then 


$$
\begin{aligned}
& \sum_{n \geq 0} t^{n} \sum_{w \in\{0,1, \ldots, k-1\}^{n}} x^{E u-\operatorname{mch}(w)} z(w)= \\
& \frac{1}{1-\left(\left(z_{0}+\cdots+z_{k-1}\right) t+\sum_{n \geq 1} t^{n(j-1)+1}(x-1)^{n} e m p_{u, n(j-1)+1}^{k}\left(z_{0}, \ldots, z_{k-1}\right)\right)}
\end{aligned}
$$

Proof. Suppose that $u \in\{0, \ldots, k-1\}^{j}$ has the minimal overlapping property and $\operatorname{red}(u)=u$. We define a ring homomorphism $\Theta$ on $\Lambda$ by letting

(1) $\Theta\left(e_{0}\right)=1$,

(2) $\Theta\left(e_{1}\right)=z_{0}+\cdots+z_{k-1}$,

(3) $\Theta\left(e_{s(j-1)+1}\right)=(-1)^{s(j-1)}(x-1)^{s} m p_{u, s(j-1)+1}^{k}\left(z_{0}, \ldots, z_{k-1}\right)$ for all $s \geq 1$, and

(4) $\Theta\left(e_{n}\right)=0$ if $n \notin\{1\} \cup\{s(j-1)+1: s \geq 1\}$.

Note that if $\Theta\left(e_{n}\right) \neq 0$ and $n \geq 1$, then the sign associated with $\Theta\left(e_{n}\right)$ is just $(-1)^{n-1}$.

We claim that for all $n \geq 1$,

$$
\Theta\left(h_{n}\right)=\sum_{w \in\{0,1, \ldots, k-1\}^{n}} x^{u-\operatorname{mch}(w)} z(w) .
$$

That is, by (19), we have that

$$
\Theta\left(h_{n}\right)=\sum_{\mu \vdash n}(-1)^{n-\ell(\mu)} B_{\mu, n} \Theta\left(e_{\mu}\right) .
$$

Now if $\mu$ is not a partition whose parts come from $\{1\} \cup\{s(j-1)+1: s \geq 1\}$, then $\Theta\left(e_{\mu}\right)=0$. Thus let $P_{j, n}$ denote the set of all partitions of $n$ whose parts come from $\{1\} \cup\{s(j-1)+1: s \geq 1\}$. For any statement $A$, let $\chi(A)=1$ if $A$ is true and $\chi(A)=0$ if $A$ is false. It follows that

$$
\begin{gathered}
\Theta\left(h_{n}\right)=\sum_{\mu \in P_{j, n}}(-1)^{n-\ell(\mu)} \sum_{\left(b_{1}, \ldots, b_{\ell(\mu)}\right) \in \mathcal{B}_{\mu, n}} \prod_{i=1}^{\ell(\mu)} \Theta\left(e_{b_{i}}\right)= \\
\sum_{\mu \in P_{j, n}}(-1)^{n-\ell(\mu)} \sum_{\left(b_{1}, \ldots, b_{\ell(\mu)}\right) \in \mathcal{B}_{\mu, n}} \prod_{i=1}^{\ell(\mu)}(-1)^{b_{i}-1}\left(\left(z_{0}+\cdots+z_{k-1}\right) \chi\left(b_{i}=1\right)+\right. \\
\sum_{\mu \in P_{j, n}} \sum_{\left(b_{1}, \ldots, b_{\ell(\mu)}\right) \in \mathcal{B}_{\mu, n}} \prod_{\left.(x-1)^{\left(b_{i}-1\right) /(j-1)} m p_{u, b_{i}}^{k}\left(z_{0}, \ldots, z_{k-1}\right) \chi\left(b_{i}>1\right)\right)=}^{\ell(\mu)}\left(\left(z_{0}+\cdots+z_{k-1}\right) \chi\left(b_{i}=1\right)+\right. \\
\left.(x-1)^{\left(b_{i}-1\right) /(j-1)} m p_{u, b_{i}}^{k}\left(\left(z_{0}, \ldots, z_{k-1}\right)\right) \chi\left(b_{i}>1\right)\right) .
\end{gathered}
$$

Next we want to give a combinatorial interpretation to the right hand side of (24). Suppose that we have a brick tabloid $B=\left(b_{1}, \ldots, b_{\ell}\right)$ of size $n$ such that $b_{i} \in\{1\} \cup\{s(j-1)+1$ : 
$s \geq 1\}$ for all $i$. Then if $b_{i}=1$, we shall interpret $z_{0}+\cdots+z_{k-1}$ as allowing us to fill $b_{i}$ with any letter from $\{0,1, \ldots, k-1\}$. If $b_{i}=s(j-1)+1>1$, then we shall interpret the term $(x-1)^{\left(b_{i}-1\right) /(j-1)} m p_{u, b_{i}}^{k}\left(z_{0}, \ldots, z_{k-1}\right)=(x-1)^{s} m p_{u, s(j-1)+1}^{k}\left(z_{0}, \ldots, z_{k-1}\right)$ as the number of ways of filling $b_{i}$ with a word $v_{i} \in \mathcal{M} \mathcal{P}_{u, s(j-1)+1}^{k}$ and then labeling each cell in $b_{i}$ which is the start of a $u$-match in $v_{i}$ with either $x$ or -1 . Let $\mathcal{O}_{u, n}$ denote the set of all labeled brick tabloids that can be constructed in this way. Thus an $O \in \mathcal{O}_{u, n}$ will consist of a triple $T=(B, w, L)$ where

1. $B=\left(b_{1}, \ldots, b_{\ell}\right)$ is a brick tabloid of shape $(n)$ such that $b_{i} \in\{1\} \cup\{s(j-1)+1$ : $s \geq 1\}$ for all $i$,

2. $w=w_{1} \ldots w_{n} \in\{0,1, \ldots, k-1\}^{n}$ is a word such that $w_{i}$ lies in $i$-th cell of $B$ for $i=1, \ldots, n$,

3. if $b_{i}=1$, then the letter in the cell corresponding to $b_{i}$ can be any letter from $\{0,1, \ldots, k-1\}$, and

4. if $b_{i}=s(j-1)+1>1$, then the cells of $b_{i}$ are filled with a word $v_{i}$ which is a maximum packing for $u$ of size $s(j-1)+1$ and each cell of $b_{i}$ which corresponds to the start of a $u$-match in $v_{i}$ is labeled with either -1 or $x$.

We then define the weight of $T, w t(T)$, to be $z(w)$ times the product of the $x$ labels in $T$ and the sign of $T, \operatorname{sign}(T)$, to be the product of the -1 labels in $T$. For example, if $k=5$ and $u=010$, then in Figure 3, we have pictured four elements of $\mathcal{O}_{010,17}$, $T_{i}=\left(B^{(i)}, w^{(i)}, L^{(i)}\right)$ for $i=1, \ldots, 4$. Then $w t\left(T^{(1)}\right)=x z_{0}^{3} z_{1}^{5} z_{2}^{2} z_{3}^{4} z_{4}^{3}, \operatorname{sign}\left(T^{(1)}\right)=1$, $\omega t\left(T^{(2)}\right)=x^{2} z_{0}^{4} z_{1}^{4} z_{2}^{2} z_{3}^{3} z_{4}^{4}, \operatorname{sign}\left(T^{(2)}\right)=1, w t\left(T^{(3)}\right)=x^{2} z_{0}^{3} z_{1}^{5} z_{2}^{2} z_{3}^{4} z_{4}^{3}, \operatorname{sign}\left(T^{(3)}\right)=1$, $w t\left(T^{(4)}\right)=x^{2} z_{0}^{3} z_{1}^{5} z_{2}^{2} z_{3}^{4} z_{4}^{3}$, and $\operatorname{sign}\left(T^{(4)}\right)=-1$. It follows that

$$
\Theta\left(h_{n}\right)=\sum_{T \in \mathcal{O}_{u, n}} \operatorname{sign}(T) w t(T) .
$$

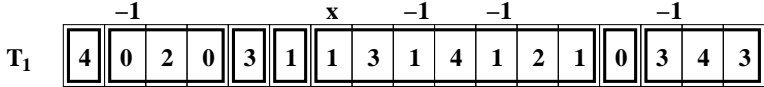

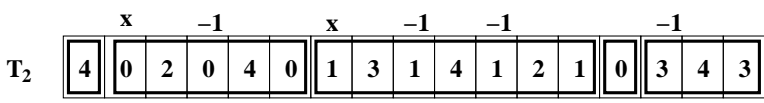

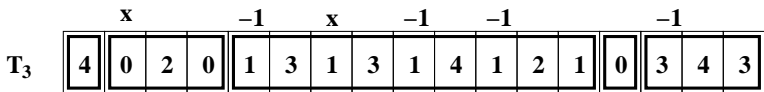

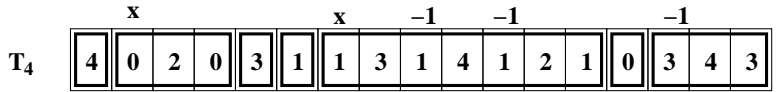

Figure 3: Elements of $\mathcal{O}_{010,17}$ 
Next we define a sign-reversing weight-preserving involution $I: \mathcal{O}_{u, n} \rightarrow \mathcal{O}_{u, n}$. If $T=(B, w, L)$, then to define $I(T)$, we scan the cells of $B=\left(b_{1}, \ldots, b_{\ell}\right)$ from left to right looking for the first time where one of the following cases hold.

Case 1. There is a brick $b_{i}$ of size $j$ whose first cell is labeled with -1 . In this case, $I(T)=\left(B^{*}, w^{*}, L^{*}\right)$ where $B^{*}$ results from $B$ by replacing the brick $b_{i}$ by $j$ bricks of size $1, w^{*}=w$, and $L^{*}$ arises from $L$ by removing the label -1 from the first cell of $b_{i}$.

Case 2. There are $j$ consecutive bricks of size 1 in $B, b_{i}, b_{i+1}, \ldots, b_{i+j-1}$ such that the letters in these cells form a $u$-match. In this case, $I(T)=\left(B^{*}, w^{*}, L^{*}\right)$ where $B^{*}$ results from $B$ by replacing the bricks $b_{i}, b_{i+1}, \ldots, b_{i+j-1}$ by a single brick $b$ of size $j, w^{*}=w$, and $L^{*}$ arises from $L$ by labeling the first cell of $b$ with a -1 .

Case 3. There is a brick $b_{i}$ of size $(s+1)(j-1)+1$ where $s \geq 1$ such that all the labels on $b_{i}$ are $x$ 's except for the cell that is $j$ cells from the right which is labeled with -1 . In this case, $I(T)=\left(B^{*}, w^{*}, L^{*}\right)$ where $B^{*}$ results from $B$ by replacing the brick $b_{i}$ by a brick of size $s(j-1)+1$ followed by $j-1$ bricks of size $1, w^{*}=w$, and $L^{*}$ arises from $L$ by removing the -1 label that was in $b_{i}$.

Case 4. There are $j$ consecutive bricks in $B, b_{i}, b_{i+1}, \ldots, b_{i+j-1}$ such that $b_{i}=s(j-1)+1>$ 1 and $b_{i+1}, \ldots b_{i+j-1}$ are of size 1 , all the labels on $b_{i}$ are $x$ 's, and the letters in these bricks form a maximum packing for $u$ of size $(s+1)(j-1)+1$. In this case, $I(T)=\left(B^{*}, w^{*}, L^{*}\right)$ where $B^{*}$ results from $B$ by replacing the bricks $b_{i}, b_{i+1}, \ldots, b_{i+j-1}$ by a single brick $b$ of size $(s+1)(j-1)+1, w^{*}=w$, and $L^{*}$ arises from $L$ by labeling the last cell of $b_{i}$ with a -1 .

Case 5. There is a brick $b_{i}$ of size $(s+1)(j-1)+1$ where $s \geq 1$ such that the first cell of $b_{i}$ is labeled with -1 . In this case, $I(T)=\left(B^{*}, w^{*}, L^{*}\right)$ where $B^{*}$ results from $B$ by replacing the brick $b_{i}$ by $j-1$ bricks of size 1 followed by a brick of size $s(j-1)+1$, $w^{*}=w$, and $L^{*}$ arises from $L$ by removing the -1 label that was on the first cell of $b_{i}$.

Case 6. There are $j$ consecutive bricks in $B, b_{i}, b_{i+1}, \ldots, b_{i+j-1}$ such that $b_{i}, \ldots b_{i+j-2}$ are bricks of size 1 and $b_{i+j-1}=s(j-1)+1>1$ and the letters in these bricks form a maximum packing for $u$ of size $(s+1)(j-1)+1$. In this case, $I(T)=\left(B^{*}, w^{*}, L^{*}\right)$ where $B^{*}$ results from $B$ by replacing the bricks $b_{i}, b_{i+1}, \ldots, b_{i+j-1}$ by a single brick $b$ of size $(s+1)(j-1)+1, w^{*}=w$, and $L^{*}$ arises from $L$ by adding a -1 label on the first cell of $b$.

Case 7. There is a brick $b_{i}$ of size $s(j-1)+1$ where $s \geq 3$ such that the first cell is labeled with an $x$ and there is a cell which has a label -1 which is not the $j$-th cell from the right. Let $t$ be the left-most cell of $b_{i}$ which is labeled with -1 . Then in this case, $I(T)=\left(B^{*}, w^{*}, L^{*}\right)$ where $B^{*}$ results from $B$ by replacing the brick $b_{i}$ by $j$ consecutive bricks $c_{1}, c_{2}, \ldots, c_{j-1}, c_{j}$ where $c_{1}$ contains all the cells of $b_{i}$ up to and including cell $t$, $c_{2}, \ldots, c_{j-1}$ are bricks of size 1 , and $c_{j}$ contains the remaining cells of $b_{i}, w=w^{*}$, and $L^{*}$ is the labeling that results from $L$ by removing the -1 label from cell $t$. 
Case 8. There are $j$ consecutive bricks $b_{i}, b_{i+1}, \ldots, b_{i+j-1}$ such that $b_{i}=c(s-1)+1>1$ and there are no -1 labels in $b_{i}, b_{i+1}, \ldots, b_{i+j-2}$ are bricks of size $1, b_{i+j-1}=d(j-$ $1)+1>1$, and the letters in these three bricks form a maximum packing for $u$ of size $(c+d+1)(j-1)+1$. In this case, $I(T)=\left(B^{*}, w^{*}, L^{*}\right)$ where $B^{*}$ results from $B$ by replacing the $j$ bricks $b_{i}, b_{i+1}, \ldots, b_{i+j-1}$ by a single brick $b, w^{*}=w$, and $L^{*}$ results from $L$ by adding a label -1 on the last cell of $b_{i}$.

If none of Cases 1-8 apply, then we set $I(T)=T$.

For example, consider images of $T_{1}, \ldots, T_{4}$ pictured in Figure 3 . It is easy to see that $T_{1}$ is in Case 1 so that $I(T)$ results by replacing the second brick by three bricks of size 1 and removing the -1 label. This results in $I\left(T_{1}\right)$ pictured in the first row of Figure 4 . It is then easy to see that $I\left(T_{1}\right)$ is in Case 2 so that $I^{2}\left(T_{1}\right)=T_{1} . T_{2}$ is in Case 3 where $b_{i}$ is the second brick. Thus we obtain $I\left(T_{2}\right)$ by replacing $b_{2}$ with a brick of size 3 followed by two bricks of size 1 and removing the -1 label from cell 4. $I\left(T_{2}\right)$ is pictured in the second row of Figure 4. It is then easy to see that $I\left(T_{2}\right)$ will be in Case 4 so that $I^{2}\left(T_{2}\right)=T_{2}$. $T_{3}$ is in Case 5 where $b_{i}$ is the third brick. Thus we obtain $I\left(T_{3}\right)$ by replacing $b_{3}$ by two bricks of size 1 followed by a brick of size 7 and removing the -1 label on cell 5. I( $\left.T_{3}\right)$ is pictured in the third row of Figure 4. It is then easy to see that $I\left(T_{3}\right)$ will be in Case 6 so that $I^{2}\left(T_{3}\right)=T_{3}$. Finally, $T_{4}$ is in Case 7 with $t=9$ so that we replace the fifth brick by three consecutive bricks of sizes 3,1 , and 3 , reading from left to right, and remove the -1 label for cell 9. $I\left(T_{4}\right)$ is pictured in the forth row of Figure 4. It is then easy to see that $I\left(T_{4}\right)$ will be in Case 8 so that $I^{2}\left(T_{4}\right)=T_{4}$.

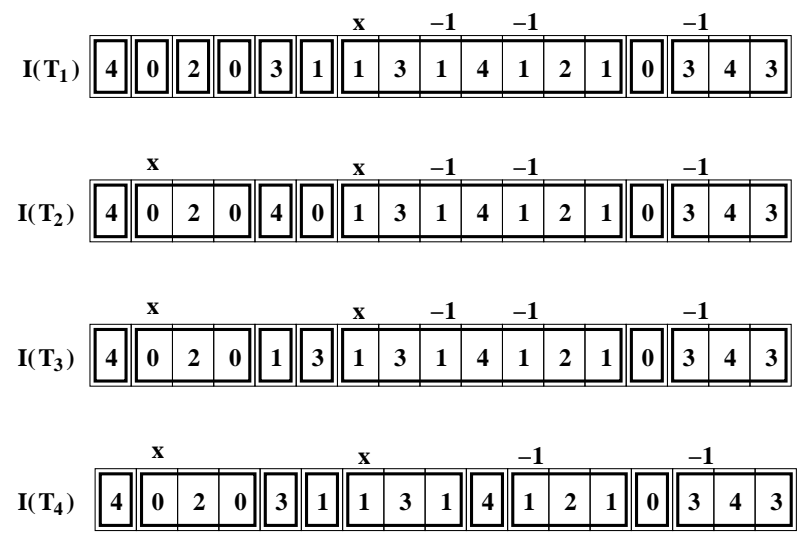

Figure 4: The images under $I$ of the elements in Figure 3.

First we claim that $I$ is an involution. For this we have to do a case by case analysis. Fix some $T=(B, w, L) \in \mathcal{O}_{u, n}$ such that $I(T) \neq T$. Let $B=\left(b_{1}, \ldots, b_{\ell}\right)$. Note that in every case, $I(T)$ is defined by changing the brick structure on some cells $s, s+1, \ldots, s+j-1$ where $w_{s} w_{s+1} \ldots w_{s+j-1}$ is a $u$-match in $w$.

First suppose that $I(T)$ was defined using Case 1 and that the brick of size $j$ that was used in the definition of $I(T)$ is $b_{i}$ and $b_{i}$ covers cells $t, t+1, \ldots, t+j-1$. Then 
in $I(T)$, we have the possibility to recombine the bricks of size 1 that now cover cells $t, t+1, \ldots, t+j-1$. Thus if $I^{2}(T) \neq T$, then it must be the case that we took some action which involved a $u$-match $w_{s} w_{s+1} \ldots w_{s+j-1}$ where $s<t$. Now it cannot be that $s+j-1<t$ since otherwise we could have taken the same action by changing the brick structure on cells $s, s+1, \ldots, s+j-1$ in $T$ which would violate the fact that we always take an action on the left-most possible cells that we can when defining $I(T)$. Because $u$ has the minimal overlapping property, the only other possibility is that $t=s+j-1$. Now if $s>1$, then the minimal overlapping property for $u$ implies that $w_{s-1} w_{s} \ldots w_{s+j-2}$ is not a $u$-match and hence the cells $s-1, s, \ldots, s+j-2$ cannot lie in a single brick since the last $j$-cells in any brick $b$ of size greater than 1 must correspond to a $u$-match in $w$. Thus it must be that in $T$, cells $s+1, \ldots, s+j-2$ must be covered by bricks of size 1 . If cell $s$ is also covered by a brick of size 1 , then we could apply Case 6 to $T$ using the $j-1$ bricks of size 1 covering cells $s, \ldots, s+j-2$ plus $b_{i}$ which would contradict the fact that for $T$, we are in Case 1 using brick $b_{i}$. If cell $s$ is part of brick $b$ of size $>1$, then we could apply Case 8 to $T$ using $b$ plus the $j-2$ bricks of size 1 covering cells $s+1, \ldots, s+j-2$ plus $b_{i}$ which again would contradict the fact that for $T$, we are in Case 1 using brick $b_{i}$. If $s=1$, then cells $s, \ldots, s+j-2$ must be covered by bricks of size 1 so that we could apply Case 6 to $T$ using the $j-1$ bricks of size 1 covering cells $s, \ldots, s+j-2$ plus $b_{i}$ which would contradict the fact that for $T$, we are in Case 1 using brick $b_{i}$. Thus the left-most $u$-match that we can use to define the image of $I$ for $I(T)$ is the $u$-match that lies in the $j$ bricks of size 1 covering cells $t, t+1, \ldots, t+j-1$ in which case we know that $I^{2}(T)=T$. An entirely similar analysis will show that if $I(T)$ is defined using Case 2, then $I^{2}(T)=T$.

Next suppose that $I(T)$ was defined by Case 3 using a brick $b_{i}$ of size $a(j-1)+1$ where $a \geq 2$ and $b_{i}$ covers cells $t, t+1, \ldots, t+a(j-1)$. Then in $I(T)$, there is a single brick $b$ covering cells $t, t+1, \ldots, t+(a-1)(j-1)$ followed by $j-1$ bricks of size 1 covering cells $t+(a-1)(j-1)+1, \ldots, t+a(j-1)$ and all the labels on $b$ are $x$ 's. In this case, if $I^{2}(T) \neq T$, then it must be the case that we took some action which involved a $u$-match $w_{s} w_{s+1} \ldots w_{s+j-1}$ where $s<t$. But then we could have taken some action by changing the brick structure on cells $s, s+1, \ldots, s+j-1$ in $T$ which would violate the fact that we always take an action on the left-most possible cells that we can when defining $I(T)$. Thus it must be the case that the left-most action that we can take to define $I$ on $I(T)$ is to combine $b$ with the $j-1$ bricks of size 1 that follow $b$ and hence $I^{2}(T)=T$. A similar analysis will show that if $I(T)$ was defined using Case 4 , then $I^{2}(T)=T$.

Next suppose that $I(T)$ was defined by Case 5 using brick $b_{i}=a(j-1)+1$ where $a \geq 2$. The analysis in this case is essentially the same as the analysis of Case 1 . That is, suppose that $b_{i}$ covers cells $t, t+1, \ldots, t+a(j-1)$. We are assuming that cell $t$ is labeled with -1 . Then in $I(T)$, the first $j-1$ cells of $b_{i}$ will be covered with bricks of size 1 and the remaining cells of $b_{i}$ with a single brick $b$. Thus if $I^{2}(T) \neq T$, then it must be the case that we took some action which involved a $u$-match $w_{s} w_{s+1} \ldots w_{s+j-1}$ where $s<t$. Now it cannot be that $s+j-1<t$ since otherwise we could have taken the same action by changing the brick structure on cells $s, s+1, \ldots, s+j-1$ in $T$ which would violate the fact that we always take an action on the left-most possible cells that we can when 
defining $I(T)$. Because $u$ has the minimal overlapping property, the only other possibility is that $t=s+j-1$. Now if $s>1$, then the minimal overlapping property for $u$ implies that $w_{s-1} w_{s} \ldots w_{s+j-2}$ is not a $u$-match and hence the cells $s-1, s, \ldots, s+j-2$ cannot lie in a single brick since the the last $j$-cells in any brick $b$ of size greater than 1 must correspond to a $u$-match in $w$. Thus it must be that in $T$, cells $s+1, \ldots, s+j-2$ must be covered by bricks of size 1 . If cell $s$ is also covered by a brick of size 1 , then we could apply Case 6 to $T$ using the $j-1$ bricks of size 1 covering cells $s, \ldots, s+j-2$ plus $b_{i}$ which would contradict the fact that for $T$, we are in Case 5 using brick $b_{i}$. If cell $s$ is part of brick $b$ of size $>1$, then we could apply Case 8 to $T$ using $b$ plus the $j-2$ bricks of size 1 covering cells $s+1, \ldots, s+j-2$ plus $b_{i}$ which again would contradict the fact that for $T$, we are in Case 5 using brick $b_{i}$. If $s=1$, then cells $s, \ldots, s+j-2$ must be covered by bricks of size 1 so that we could apply Case 6 to $T$ using the $j-1$ bricks of size 1 covering cells $s, \ldots, s+j-2$ plus $b_{i}$ which would contradict the fact that for $T$, we are in Case 5 using brick $b_{i}$. Thus the left-most $u$-match that we can use to define the image of $I$ for $I(T)$ is the $u$-match that lies $j-1$ bricks of size 1 covering cells $t, t+1, \ldots, t+j-1$ plus the brick $b$ in which case we know that $I^{2}(T)=T$. An entirely similar analysis will show that if $I(T)$ is defined using Case 6, then $I^{2}(T)=T$.

Finally suppose that $I(T)$ was defined using Case 7 using a brick $b_{i}$ of size $a(j-1)+1$ where $a \geq 3$. Suppose that $b_{i}$ covers cells $t, t+1, \ldots, t+a(j-1)$. We are assuming that cell $t$ has label $x$ and that the left-most cell of $b_{i}$ which is labeled with -1 occurs on cell $t+b(j-1)$ where $1 \leq b<a-1$. Then in $I(T)$ there is a single brick $b^{*}$ covering cells $t, t+1, \ldots, t+b(j-1)$ followed by $j-2$ bricks of size 1 covering cells $t+b(j-1)+1, \ldots, t+b(j-1)+j-2$ followed by a brick $b^{* *}$ covering the remaining cells of $b_{i}$. Moreover all the labels on $b^{*}$ are $x$ 's. In this case, if $I^{2}(T) \neq T$, then it must be the case that we took some action which involved a $u$-match $w_{s} w_{s+1} \ldots w_{s+j-1}$ where $s<t$. But then we could have taken some action by changing the brick structure on cells $s, s+1, \ldots, s+j-1$ in $T$ which would violate the fact that we always take an action on the left-most possible cells that we can when defining $I(T)$. Thus it must be the case that the left-most action that we can take to define is to recombine $b^{*}$ plus the following $j-2$ bricks of size 1 plus $b^{* *}$ into a single brick so that $I^{2}(T)=T$. An entirely similar analysis will show that if $I(T)$ is defined using Case 8, then $I^{2}(T)=T$.

It is easy to see that if $I(T) \neq T$, then $\operatorname{sign}(T) w t(T)=-\operatorname{sign}(I(T)) w t(I(T))$. Hence $I$ shows that

$$
\Theta\left(h_{n}\right)=\sum_{T \in \mathcal{O}_{u, n}, I(T)=T} \operatorname{sign}(T) w t(T) .
$$

Thus we must examine the fixed points of $I$. Suppose that $T=(B, w, L)$ is a fixed point of $I$ where $B=\left(b_{1}, \ldots, b_{\ell}\right)$. There cannot be any -1 labels on any of the bricks in $B$ since otherwise we could apply one of Cases $1,3,5$, or 7 . Thus if $I(T)=T, \operatorname{sign}(T)=1$. It follows that $w t(T)=x^{c} z(w)$ where $c$ is the number of $u$-matches in $w$ that lie entirely with in some brick $b_{i}$ in $B$. We claim that any $u$-match in $w$ must lie entirely within some brick. That is, suppose that $w=w_{1} \ldots w_{n}$ and $w_{s} w_{s+1} \ldots w_{s+j-1}$ a $u$-match that does not lie in a single brick. Because $u$ has the minimal overlapping property, there are only four possibilities, namely, 
(i) cells $s, s+1, \ldots, s+j-1$ are covered by bricks of size 1 ,

(ii) cell $s$ is part of brick $b_{i}$ of size $>1$ and cells $s+1, \ldots, s+j-1$ are covered by bricks of size 1 ,

(iii) cell $s+j-1$ is part of brick $b_{i}$ of size $>1$ and cells $s, \ldots, s+j-2$ are covered by bricks of size 1 , or

(iv) cell $s$ is part of a brick $b_{i}$ of size $>1 b_{i+1}, \ldots, b_{i+j-2}$ are bricks of size 1 covering cells $s+1, \ldots, s+j-2$, and cell $s+j-1$ is part of brick $b_{i+j-1}$ which is of size $>1$.

In case (i), we could apply Case 2 of the definition of $I$ to cells $s, s+1, \ldots, s+j-1$. In case (ii), we could apply Case 4 of the definition of $I$ to cells of $b_{i}$ plus cells $s+1, \ldots, s+j-1$. In case (iii), we could apply Case 6 of the definition of $I$ to cells $s, \ldots, s+j-2$ plus the cells of $b_{i}$. In case (iv), we can apply Case 8 of the definition of $I$ to the cells of contained in the bricks $b_{i}, \ldots, b_{i+j-1}$. Thus in all the cases (i)-(iv), it would be the case that $I(T) \neq T$ which contradicts our choice of $T$. Thus we have shown that if $I(T)=T$, then $\operatorname{sign}(T) w t(T)=x^{u-\operatorname{mch}(w)} z(w)$. Finally note that if $w \in\{0, \ldots, k-1\}^{n}$, then we can construct a fixed point of $I$ by placing bricks which cover the maximal length maximum packings in $w$, covering the remaining cells by bricks of size 1, and labeling the start of each $u$-match in $w$ by $x$. It thus follows that

$$
\Theta\left(h_{n}\right)=\sum_{w \in\{0, \ldots, k-1\}^{n}} x^{u-\operatorname{mch}(w)} z(w) .
$$

But then

$$
\begin{aligned}
& \Theta(H(t))= \sum_{n \geq 0} t^{n} \sum_{w \in\{0, \ldots, k-1\}^{n}} x^{u-\operatorname{mch}(w)} z(w)=\Theta(1 / E(-t))= \\
& \frac{1}{1+\sum_{n \geq 1}(-t)^{n} \Theta\left(e_{n}\right)}= \\
& \frac{1}{1-\left(\left(z_{0}+\cdots+z_{k-1}\right) t+\sum_{n \geq 1} t^{n(j-1)+1}(x-1)^{n} m p_{u, n(j-1)+1}^{k}\left(z_{0}, \ldots, z_{k-1}\right)\right)}
\end{aligned}
$$

which proves $(20)$.

If we replace $u$-matchings by exact $u$-matching throughout the proof of $(20)$, then we will get a proof of (21).

Next we prove our main results for $k$-colored permutations.

Theorem 6. (III) If $(\tau, u) \in C_{k} \curlywedge S_{j}, \operatorname{red}(u)=u$, and $(\tau, u)$ has the $C_{k} \prec S_{n}$-minimal overlapping property, then

$$
\begin{gathered}
\sum_{n \geq 0} \frac{t^{n}}{n !} \sum_{(\sigma, w) \in C_{k} l S_{n}} x^{(\tau, u)-\operatorname{mch}((\sigma, u))} p^{\operatorname{coinv}(\sigma)} q^{\operatorname{inv}(\sigma)} z(w)= \\
\frac{1}{1-\left(\left(z_{0}+\cdots+z_{k-1}\right) t+\sum_{n \geq 1} \frac{t^{n(j-1)+1}}{[n(j-1)+1]_{p, q} !}(x-1)^{n} m p_{(\tau, u), n(j-1)+1}^{k}\left(p, q, z_{0}, \ldots, z_{k-1}\right)\right)} .
\end{gathered}
$$


(IV) If $(\tau, u) \in C_{k} 2 S_{j}$ has the $C_{k} \imath S_{n}$-exact match minimal overlapping property, then

$$
\begin{gathered}
\sum_{n \geq 0} \frac{t^{n}}{n !} \sum_{(\sigma, w) \in C_{k} \imath S_{n}} x^{(\tau, E u)-\operatorname{mch}((\sigma, u))} p^{\operatorname{coinv}(\sigma)} q^{\operatorname{inv}(\sigma)} z(w)= \\
\frac{1}{1-\left(\left(z_{0}+\cdots+z_{k-1}\right) t+\sum_{n \geq 1} \frac{t^{n(j-1)+1}}{[n(j-1)+1]_{q} !}(x-1)^{n} e m p_{(\tau, u), n(j-1)+1}^{k}\left(p, q, z_{0}, \ldots, z_{k-1}\right)\right)} .
\end{gathered}
$$

Proof. We shall see that the proof of Theorem 6 is very similar to the proof of Theorem 5 . Suppose that $(\tau, u) \in C_{k} 2 S_{j}$ has the $C_{k} 2 S_{n}$-minimal overlapping property and $\operatorname{red}(u)=u$. We define a ring homomorphism $\Gamma$ on $\Lambda$ by letting

(1) $\Gamma\left(e_{0}\right)=1$

(2) $\Gamma\left(e_{1}\right)=z_{0}+\cdots+z_{k-1}$,

(3) $\Gamma\left(e_{s(j-1)+1}\right)=(-1)^{s(j-1)}(x-1)^{s} \frac{1}{[s(j-1)+1]_{p, q} !} m p_{(\tau, u), s(j-1)+1}^{k}\left(p, q, z_{0}, \ldots, z_{k-1}\right)$ for all $s \geq 1$, and

(4) $\Gamma\left(e_{n}\right)=0$ if $n \notin\{1\} \cup\{s(j-1)+1: s \geq 1\}$.

Note that if $\Gamma\left(e_{n}\right) \neq 0$ and $n \geq 1$, then the sign associated with $\Gamma\left(e_{n}\right)$ is just $(-1)^{n-1}$.

We claim that for all $n \geq 1$,

$$
[n]_{p, q} ! \Gamma\left(h_{n}\right)=\sum_{(\sigma, w) \in C_{k} S_{n}} p^{\operatorname{coinv}(\sigma)} q^{\operatorname{inv}(\sigma)} x^{(\tau, u)-\operatorname{mch}(w)} z(w) .
$$

That is, by (19), we have that

$$
[n]_{p, q} ! \Gamma\left(h_{n}\right)=[n]_{p, q} ! \sum_{\mu \vdash n}(-1)^{n-\ell(\mu)} B_{\mu, n} \Gamma\left(e_{\mu}\right) .
$$

Now if $\mu$ is not a partition whose parts come from $\{1\} \cup\{s(j-1)+1: s \geq 1\}$, then $\Gamma\left(e_{\mu}\right)=0$. Thus let $P_{j, n}$ denote the set of all partitions of $n$ whose parts come from $\{1\} \cup\{s(j-1)+1: s \geq 1\}$. It follows that

$$
\begin{gathered}
{[n]_{p, q} ! \Gamma\left(h_{n}\right)=[n]_{p, q} ! \sum_{\mu \in P_{j, n}}(-1)^{n-\ell(\mu)} \sum_{\left(b_{1}, \ldots, b_{\ell(\mu)}\right) \in \mathcal{B}_{\mu, n}} \prod_{i=1}^{\ell(\mu)} \Gamma\left(e_{b_{i}}\right)=} \\
{[n]_{p, q} ! \sum_{\mu \in P_{j, n}}(-1)^{n-\ell(\mu)} \sum_{\left(b_{1}, \ldots, b_{\ell(\mu)}\right) \in \mathcal{B}_{\mu, n}} \prod_{i=1}^{\ell(\mu)}(-1)^{b_{i}-1}\left(\left(z_{0}+\cdots+z_{k-1}\right) \chi\left(b_{i}=1\right)+\right.} \\
\left.(x-1)^{\left(b_{i}-1\right) /(j-1)} \frac{1}{\left[b_{i}\right]_{p, q} !} m p_{(\tau, u), b_{i}}^{k}\left(p, q, z_{0}, \ldots, z_{k-1}\right) \chi\left(b_{i}>1\right)\right)= \\
\sum_{\mu \in P_{j, n}\left(b_{1}, \ldots, b_{\ell(\mu)}\right) \in \mathcal{B}_{\mu, n}} p^{\sum_{i=1}^{\ell(\mu)}\left(\begin{array}{c}
\left.b_{i}\right) \\
2
\end{array}\right)\left[\begin{array}{c}
\ell(\mu) \\
b_{1}, \ldots, b_{\ell(\mu)}
\end{array}\right]} \prod_{p, q}\left(\left(z_{0}+\cdots+z_{k-1}\right) \chi\left(b_{i}=1\right)+\right.
\end{gathered}
$$




$$
\left.(x-1)^{\left(b_{i}-1\right) /(j-1)} p^{-\left(\begin{array}{c}
b_{i} \\
2
\end{array}\right)} m p_{(\tau, u), b_{i}}^{k}\left(p, q, z_{0}, \ldots, z_{k-1}\right) \chi\left(b_{i}>1\right)\right) .
$$

Next we want to give a combinatorial interpretation to the right hand side of (31). Suppose that we have a brick tabloid $B=\left(b_{1}, \ldots, b_{\ell}\right)$ of size $n$ such that $b_{i} \in\{1\} \cup\{s(j-1)+1$ : $s \geq 1\}$ for all $i$. By Lemma 4 , we can interpret $p^{\sum_{i=1}^{\ell(\mu)}\left(\begin{array}{c}b_{i} \\ 2\end{array}\right)}\left[\begin{array}{c}n \\ b_{1}, \ldots, b_{\ell(\mu)}\end{array}\right]$ as the ways of filling the cells of $B$ with a permutation $\alpha$ such that $\alpha$ is increasing in each brick of $B$ and we weight $\alpha$ by $p^{\operatorname{coinv}(\alpha)} q^{\operatorname{inv}(\alpha)}$. Then if $b_{i}=1$, we shall interpret $z_{0}+\cdots+z_{k-1}$ as allowing us to place any letter from $\{0,1, \ldots, k-1\}$ on top of the integer from $\alpha$ that is in brick $b_{i}$. If $b_{i}=s(j-1)+1>1$, then let $1 \leq a_{1}^{i}<\ldots<a_{s(j-1)+1}^{i} \leq n$ be the elements of $\alpha$ which are in the cells of $b_{i}$ reading from left to right. We shall interpret the term

$$
\begin{aligned}
& (x-1)^{\left(b_{i}-1\right) /(j-1)} p^{-\left(\begin{array}{c}
b_{i} \\
2
\end{array}\right)} m p_{(\tau, u), b_{i}}\left(p, q, z_{0}, \ldots, z_{k-1}\right)= \\
& (x-1)^{s} p^{-\left(\begin{array}{c}
b_{i} \\
2
\end{array}\right)} m p_{(\tau, u), s(j-1)+1}\left(p, q, z_{0}, \ldots, z_{k-1}\right)
\end{aligned}
$$

as the number of ways of filling $b_{i}$ with a pair $\left(\beta_{i}, v_{i}\right) \in \mathcal{M P}_{(\tau, u), s(j-1)+1}^{k}$ and then labeling each cell in $b_{i}$ which is the start of $(\tau, u)$-match in $\left(\beta_{i}, v_{i}\right)$ with either $x$ or -1 . In this case, we weight $\left(\beta_{i}, v_{i}\right)$ by $p^{-\left(\begin{array}{c}b_{i} \\ 2\end{array}\right)} p^{\operatorname{coinv}\left(\beta_{i}\right)} q^{\operatorname{inv}\left(\beta_{i}\right)} z\left(v_{i}\right)$. Finally, we replace the numbers by $1, \ldots, s(j-1)+1$ that occur in $\beta_{i}$ by $a_{1}, \ldots, a_{s(j-1)+1}$ respectively. Doing this for each brick will result in a filling of the cells of $B$ with a pair $(\sigma, w) \in C_{k} \backslash S_{n}$. We claim that that $\operatorname{coinv}(\alpha)-\sum_{b_{i}>1}\left(\begin{array}{c}b_{i} \\ 2\end{array}\right)+\operatorname{coinv}\left(\beta_{i}\right)=\operatorname{coinv}(\sigma)$. That is, $\operatorname{coinv}(\alpha)$ accounts for the coinversions that come from pairs of elements that lie in two different bricks plus $\sum_{b_{i}>1}\left(\begin{array}{c}b_{i} \\ 2\end{array}\right)$ which accounts for the coinversions that come from pairs that lie in the same brick. Thus coinv $(\alpha)-\sum_{b_{i}>1}\left(\begin{array}{c}b_{i} \\ 2\end{array}\right)$ counts only the coinversions that come from pairs of elements in $\sigma$ that lie in two different bricks. Then $\sum_{b_{i}>1} \operatorname{coinv}\left(\beta_{i}\right)$ accounts for the coinversions that come from pairs of $\sigma$ that lie in the same brick. A similar argument will show that $\operatorname{inv}(\alpha)+\sum_{b_{i}>1} \operatorname{inv}\left(\beta_{i}\right)=\operatorname{inv}(\sigma)$. Thus the final weight of $(\sigma, w)$ will be $p^{\operatorname{coinv}(\sigma)} q^{\operatorname{inv}(\sigma)} z(w)$.

Let $\mathcal{Q}_{(\tau, u), n}$ denote the set of all labeled brick tabloids that can be constructed in this way. Thus a $Q \in \mathcal{Q}_{(\tau, u), n}$ will consist of a triple $T=(B,(\sigma, w), L)$ where $\sigma=\sigma_{1} \ldots \sigma_{n} \in$ $S_{n}, w=w_{1} \ldots w_{n} \in\{0,1, \ldots, k-1\}^{n}$, and

1. $B=\left(b_{1}, \ldots, b_{\ell}\right)$ is a brick tabloid of shape $(n)$ such that $b_{i} \in\{1\} \cup\{s(j-1)+1$ : $s \geq 1\}$ for all $i$,

2. $(\sigma, w) \in C_{k} 2 S_{n}$ is a $k$-colored permutation,

3. if $b_{i}=1$ and covers cell $t$, then $\left(\sigma_{t}, w_{t}\right)$ is allowed to be any pair from $\{1, \ldots, n\} \times$ $\{0,1, \ldots, k-1\}$, and

4. if $b_{i}=s(j-1)+1>1$, then cells of $b_{i}$ are filled with a pair $\left(\gamma_{i}, v_{i}\right)$ such that $\left(\operatorname{pred}\left(\gamma_{i}\right), v_{i}\right)$ is a maximum packing for $(\tau, u)$ of size $s(j-1)+1$ and each cell of $b_{i}$ which corresponds to the start of $(\tau, u)$-match in $\left(\gamma_{i}, v_{i}\right)$ is labeled with either -1 or $x$. 
We then define the weight of $T, w t(T)$, to be $p^{\operatorname{coinv}(\sigma)} q^{\operatorname{inv}(\sigma)} z(w)$ times the product of the $x$ labels in $T$ and the sign of $T, \operatorname{sign}(T)$, to be the product of the -1 labels in $T$. For example, if $k=5$ and $(\tau, u)=(213,010)$, then in Figure 5 we have pictured four elements of $\mathcal{Q}_{(213,010), 17}, T_{i}=\left(B^{(i)},\left(\sigma^{(i)}, w^{(i)}\right), L^{(i)}\right)$ for $i=1, \ldots, 4$. Then $w t\left(T^{(1)}\right)=x z_{0}^{3} z_{1}^{5} z_{2}^{2} z_{3}^{4} z_{4}^{3} p^{65} q^{71}, \operatorname{sign}\left(T^{(1)}\right)=1$, $w t\left(T^{(2)}\right)=x^{2} z_{0}^{4} z_{1}^{4} z_{2}^{2} z_{3}^{3} z_{4}^{4} p^{65} q^{71}, \operatorname{sign}\left(T^{(2)}\right)=1$, $w t\left(T^{(3)}\right)=x^{2} z_{0}^{3} z_{1}^{5} z_{2}^{2} z_{3}^{4} z_{4}^{3} p^{66} q^{70}, \operatorname{sign}\left(T^{(3)}\right)=1$, $w t\left(T^{(4)}\right)=x^{2} z_{0}^{3} z_{1}^{5} z_{2}^{2} z_{3}^{4} z_{4}^{3} p^{66} q^{70}$, and $\operatorname{sign}\left(T^{(4)}\right)=1$. It follows that

$$
[n]_{p, q} ! \Gamma\left(h_{n}\right)=\sum_{T \in \mathcal{Q}_{(\tau, u), n}} \operatorname{sign}(T) w t(T)
$$

\begin{tabular}{|c|c|c|c|c|c|c|c|c|c|c|c|c|c|c|c|c|c|}
\hline & & -1 & & & & & $\mathbf{x}$ & & -1 & & -1 & & & & -1 & & \\
\hline T & \begin{tabular}{c||}
4 \\
1
\end{tabular} & $\begin{array}{c}\mathbf{0} \\
\mathbf{1 0}\end{array}$ & $\begin{array}{l}2 \\
9\end{array}$ & $\begin{array}{c}0 \\
11\end{array}$ & $\begin{array}{l}3 \\
5\end{array}$ & $\mid$\begin{tabular}{||c}
1 \\
12
\end{tabular} & $\begin{array}{l}1 \\
4\end{array}$ & $\begin{array}{l}3 \\
2\end{array}$ & $\begin{array}{l}\mathbf{1} \\
\mathbf{8}\end{array}$ & $\begin{array}{l}4 \\
1\end{array}$ & $\begin{array}{c}1 \\
13\end{array}$ & $\begin{array}{l}2 \\
6\end{array}$ & $\begin{array}{c}1 \\
16\end{array}$ & \begin{tabular}{|c||}
0 \\
15
\end{tabular} & $\begin{array}{l}3 \\
7\end{array}$ & $\begin{array}{l}4 \\
3\end{array}$ & \begin{tabular}{r|}
3 \\
14
\end{tabular} \\
\hline & & $\mathbf{x}$ & & -1 & & & $\mathbf{x}$ & & -1 & & -1 & & & & -1 & & \\
\hline r & \begin{tabular}{|c||c||}
4 \\
17
\end{tabular} \mid & \begin{tabular}{c|c}
$\mathbf{0}$ \\
$\mathbf{1 0}$
\end{tabular} & $\begin{array}{l}2 \\
9\end{array}$ & $\begin{array}{c}0 \\
11\end{array}$ & $\begin{array}{l}4 \\
5\end{array}$ & $\begin{array}{r}0 \\
12\end{array}$ & $\begin{array}{l}1 \\
4\end{array}$ & $\begin{array}{l}3 \\
2\end{array}$ & $\begin{array}{l}1 \\
8\end{array}$ & $\begin{array}{l}\mathbf{4} \\
1\end{array}$ & $\begin{array}{c}1 \\
13\end{array}$ & $\begin{array}{l}2 \\
6\end{array}$ & \begin{tabular}{c||c}
10 \\
16
\end{tabular} & $\mid$\begin{tabular}{c|c}
0 \\
15
\end{tabular} & $\begin{array}{l}3 \\
7\end{array}$ & $\begin{array}{l}4 \\
3\end{array}$ & \begin{tabular}{|c||}
3 \\
14
\end{tabular} \\
\hline & & $\mathbf{x}$ & & & -1 & & $\mathbf{x}$ & & -1 & & -1 & & & & -1 & & \\
\hline & \begin{tabular}{|c||c|}
4 \\
17
\end{tabular} \mid & $\begin{array}{c}\mathbf{0} \\
\mathbf{1 0}\end{array}$ & $\begin{array}{l}2 \\
9\end{array}$ & \begin{tabular}{c||}
0 \\
11
\end{tabular} & $\begin{array}{l}1 \\
5\end{array}$ & $\begin{array}{l}3 \\
4\end{array}$ & $\begin{array}{l}1 \\
8\end{array}$ & $\begin{array}{l}3 \\
2\end{array}$ & $\begin{array}{c}1 \\
12\end{array}$ & $\begin{array}{l}4 \\
1\end{array}$ & $\begin{array}{c}1 \\
13\end{array}$ & $\begin{array}{l}2 \\
6\end{array}$ & \begin{tabular}{c||}
1 \\
16
\end{tabular} & $\left|\begin{array}{c}0 \\
15\end{array}\right|$ & \begin{tabular}{l|}
3 \\
7
\end{tabular} & $\begin{array}{l}4 \\
3\end{array}$ & \begin{tabular}{r|}
3 \\
14
\end{tabular} \\
\hline & & $\mathbf{x}$ & & & $\mathbf{x}$ & & -1 & & -1 & & -1 & & & & -1 & & \\
\hline & $\begin{array}{c}4 \\
17\end{array}$ & $\begin{array}{c}\mathbf{0} \\
\mathbf{1 0}\end{array}$ & $\begin{array}{l}2 \\
9\end{array}$ & $\begin{array}{c}0 \\
11\end{array}$ & $\begin{array}{l}1 \\
5\end{array}$ & $\begin{array}{l}3 \\
4\end{array}$ & $\begin{array}{l}1 \\
8\end{array}$ & $\begin{array}{l}3 \\
2\end{array}$ & $\begin{array}{c}1 \\
12\end{array}$ & $\begin{array}{l}4 \\
1\end{array}$ & $\begin{array}{c}1 \\
13\end{array}$ & $\begin{array}{l}2 \\
6\end{array}$ & \begin{tabular}{c||}
1 \\
16
\end{tabular} & \begin{tabular}{|c||}
0 \\
15
\end{tabular} & $\begin{array}{l}3 \\
7\end{array}$ & $\begin{array}{l}\mathbf{4} \\
\mathbf{3}\end{array}$ & \begin{tabular}{|c||}
3 \\
14
\end{tabular} \\
\hline
\end{tabular}

Figure 5: Elements of $\mathcal{Q}_{(213,010), 17}$.

Next we define a sign-reversing weight-preserving involution $I: \mathcal{Q}_{(\tau, u), n} \rightarrow \mathcal{Q}_{(\tau, u), n}$ in essentially the same way that we did in Theorem 5 . That is, if $T=(B,(\sigma, w), L)$, then to define $I(T)$, we scan the cells of $B=\left(b_{1}, \ldots, b_{\ell}\right)$ from left to right looking for the first time we are in one of the following cases.

Case 1. There is a brick $b_{i}$ of size $j$ whose first cell is labeled with -1 . In this case, $I(T)=\left(B^{*},\left(\sigma^{*} w^{*}\right), L^{*}\right)$ where $B^{*}$ results from $B$ by replacing the brick $b_{i}$ by $j$ bricks of size $1,\left(\sigma^{*}, w^{*}\right)=(\sigma, w)$, and $L^{*}$ arises from $L$ by removing the label -1 from the first cell of $b_{i}$.

Case 2. There are $j$ consecutive bricks of size 1 in $B, b_{i}, b_{i+1}, \ldots, b_{i+j-1}$ such that the letters in these cells form a $(\tau, u)$-match. In this case, $I(T)=\left(B^{*},\left(\sigma^{*}, w^{*}\right), L^{*}\right)$ where $B^{*}$ results from $B$ by replacing the bricks $b_{i}, b_{i+1}, \ldots, b_{i+j-1}$ by a single brick $b$ of size $j$, $\left(\sigma^{*}, w^{*}\right)=(\sigma, w)$, and $L^{*}$ arises from $L$ by labeling the first cell of $b$ with a -1 .

Case 3. There is a brick $b_{i}$ of size $(s+1)(j-1)+1$ where $s \geq 1$ such that all the 
labels on $b_{i}$ are $x$ 's except for the cell that is $j$ cells from the right which is labeled with -1 . In this case, $I(T)=\left(B^{*},\left(\sigma^{*}, w^{*}\right), L^{*}\right)$ where $B^{*}$ results from $B$ by replacing the brick $b_{i}$ by a brick of size $s(j-1)+1$ followed by $j-1$ bricks of size $1,\left(\sigma^{*}, w^{*}\right)=(\sigma, w)$, and $L^{*}$ arises from $L$ by removing the -1 label that was in $b_{i}$.

Case 4. There are $j$ consecutive bricks in $B, b_{i}, b_{i+1}, \ldots, b_{i+j-1}$ such that $b_{i}=s(j-1)+1>1$ and $b_{i+1}, \ldots b_{i+j-1}$ are of size 1 , all the labels on $b_{i}$ are $x$ 's, and the letters in these bricks form a maximum packing for $(\tau, u)$ of size $(s+1)(j-1)+1$. In this case, $I(T)=\left(B^{*},\left(\sigma^{*}, w^{*}\right), L^{*}\right)$ where $B^{*}$ results from $B$ by replacing the bricks $b_{i}, b_{i+1}, \ldots, b_{i+j-1}$ by a single brick $b$ of size $(s+1)(j-1)+1,\left(\sigma^{*}, w^{*}\right)=(\sigma, w)$, and $L^{*}$ arises from $L$ by labeling the last cell of $b_{i}$ with a -1 .

Case 5. There is a brick $b_{i}$ of size $(s+1)(j-1)+1$ where $s \geq 1$ such that the first cell of $b_{i}$ is labeled with -1 . In this case, $I(T)=\left(B^{*},\left(\sigma^{*}, w^{*}\right), L^{*}\right)$ where $B^{*}$ results from $B$ by replacing the brick $b_{i}$ by $j-1$ bricks of size 1 followed by a brick of size $s(j-1)+1$, $\left(\sigma^{*}, w^{*}\right)=(\sigma, w)$, and $L^{*}$ arises from $L$ by removing the -1 label that was on the first cell of $b_{i}$.

Case 6. There are $j$ consecutive bricks in $B, b_{i}, b_{i+1}, \ldots, b_{i+j-1}$ such that $b_{i}, \ldots b_{i+j-2}$ are of size 1 and $b_{i+j-1}=s(j-1)+1>1$ and the letters in these bricks form a maximum packing for $(\tau, u)$ of size $(s+1)(j-1)+1$. In this case, $I(T)=\left(B^{*},\left(\sigma^{*}, w^{*}\right), L^{*}\right)$ where $B^{*}$ results from $B$ by replacing the bricks $b_{i}, b_{i+1}, \ldots, b_{i+j-1}$ by a single brick $b$ of size $(s+1)(j-1)+1,\left(\sigma^{*}, w^{*}\right)=(\sigma, w)$, and $L^{*}$ arises from $L$ by labeling the first cell of $b$ with a -1 .

Case 7. There is a brick $b_{i}$ of size $s(j-1)+1$ where $s \geq 3$ such that the first cell is labeled with an $x$ and there is a cell which has a label -1 which is not the $j$-th cell from the right. Let $t$ be the left-most cell of $b_{i}$ which is labeled with -1 . Then in this case, $I(T)=\left(B^{*},\left(\sigma^{*}, w^{*}\right), L^{*}\right)$ where $B^{*}$ results from $B$ by replacing the brick $b_{i}$ by $j$ consecutive bricks $c_{1}, c_{2}, \ldots, c_{j-1}, c_{j}$ where $c_{1}$ contains all the cells of $b_{i}$ up to and including cell $t$, $c_{2}, \ldots, c_{j-1}$ are bricks of size 1 , and $c_{j}$ contains the remaining cells of $b_{i},\left(\sigma^{*}, w^{*}\right)=(\sigma, w)$, and $L^{*}$ is the labeling that results from $L$ by removing the -1 label from cell $t$.

Case 8. There are $j$ consecutive bricks $b_{i}, b_{i+1}, \ldots, b_{i+j-1}$ such that $b_{i}=c(s-1)+1>1$ and has no -1 labels, $b_{i+1}, \ldots, b_{i+j-2}$ are bricks of size $1, b_{i+j-1}=d(j-1)+1>1$, and the letters in these three bricks form a maximum packing for $(\tau, u)$ of size $(c+d+1)(j-1)+1$. In this case, $I(T)=\left(B^{*},\left(\sigma^{*}, w^{*}\right), L^{*}\right)$ where $B^{*}$ results from $B$ by replacing the $j$ bricks $b_{i}, b_{i+1}, \ldots, b_{i+j-1}$ by a single brick $b,\left(\sigma^{*}, w^{*}\right)=(\sigma, w)$, and $L^{*}$ results from $L$ by adding a label -1 on the last cell of $b_{i}$.

If none of Cases 1-8 apply, then we set $I(T)=T$.

For example, the images of $T_{1}, \ldots, T_{4}$ as pictured in Figure 5 are pictured in Figure 6. As in Theorem $5, I$ is a sign-reversing weight-preserving involution. Hence $I$ shows 


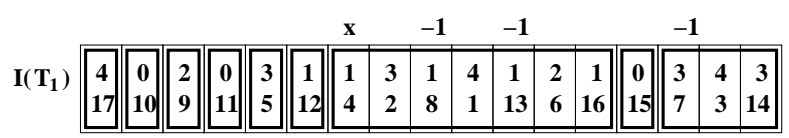

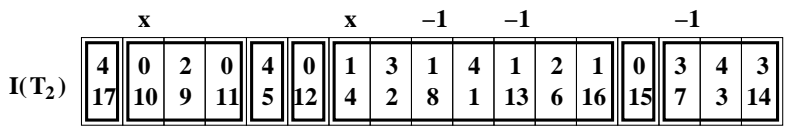

\begin{tabular}{|c|c|c|c|c|c|c|c|c|c|c|c|c|c|c|c|c|c|}
\hline \multirow[b]{3}{*}{$\mathbf{I}\left(\mathbf{T}_{\mathbf{3}}\right)$} & \multicolumn{4}{|c|}{$\mathbf{x}$} & & & \multicolumn{2}{|l|}{$\mathbf{x}$} & \multicolumn{2}{|c|}{-1} & \multicolumn{2}{|l|}{-1} & \multicolumn{5}{|c|}{-1} \\
\hline & 4 & 0 & 2 & $\mathbf{0}$ & 1 & 3 & 1 & 3 & 1 & 4 & 1 & 2 & 1 & 0 & 3 & 4 & 3 \\
\hline & 17 & 10 & 9 & 11 & 5 & 4 & 8 & 2 & 12 & 1 & 13 & 6 & 16 & 15 & 7 & 3 & 14 \\
\hline
\end{tabular}

\begin{tabular}{|c|c|c|c|c|c|c|c|c|c|c|c|c|c|c|c|c|c|}
\hline \multirow{3}{*}{$\mathbf{I}\left(\mathbf{T}_{\mathbf{4}}\right)$} & \multicolumn{2}{|r|}{$\mathbf{x}$} & & \multicolumn{3}{|c|}{$\mathbf{x}$} & & \multicolumn{3}{|c|}{-1} & \multicolumn{2}{|c|}{-1} & & \multicolumn{4}{|c|}{-1} \\
\hline & 4 & 0 & 2 & 0 & 1 & 3 & 1 & 3 & 1 & 4 & 1 & 2 & 1 & 0 & 3 & 4 & 3 \\
\hline & 17 & 10 & 9 & 11 & 5 & 4 & 8 & 2 & 12 & 1 & 13 & 6 & 16 & 15 & 7 & 3 & 14 \\
\hline
\end{tabular}

Figure 6: The images under $I$ of the elements in Figure 5.

that

$$
[n]_{p, q} ! \Gamma\left(h_{n}\right)=\sum_{T \in \mathcal{Q}_{(\tau, u), n}, I(T)=T} \operatorname{sign}(T) w t(T) .
$$

Thus we must examine the fixed points of $I$. We can argue exactly as in Theorem 1 that if $I(T)=T$ where $T=(B,(\sigma, w), L)$, then $\operatorname{sign}(T)=1$ since there can be no -1 labels and $w t(T)=x^{(\tau, u)-\operatorname{mch}((\sigma, w))} p^{\operatorname{coinv}(\sigma)} q^{\operatorname{inv}(\sigma)} z(w)$. Moreover if $(\sigma, w) \in C_{k} 2 S_{n}$, then we can construct a fixed point of $I$ by placing bricks which cover the maximal length maximum packings for $(\tau, u)$ in $(\sigma, w)$, covering the remaining cells by bricks of size 1 , and labeling the start of each $(\tau, u)$-match in $w$ by $x$. It thus follows that

$$
[n]_{p, q} ! \Gamma\left(h_{n}\right)=\sum_{(\sigma, w) \in C_{k} 2 S_{n}} x^{(\tau, u)-\operatorname{mch}((\sigma, w))} p^{\operatorname{coinv}(\sigma)} q^{\operatorname{inv}(\sigma)} z(w) .
$$

But then,

$$
\begin{gathered}
\Gamma(H(t))=\sum_{n \geq 0} \frac{t^{n}}{[n]_{p, q} !} \sum_{(\sigma, w) \in C_{k} l S_{n}} x^{(\tau, u)-\operatorname{mch}((\sigma, w))} p^{\operatorname{coinv}(\sigma)} q^{\operatorname{inv}(\sigma)} z(w)=\Gamma(1 / E(-t))= \\
\frac{1}{1+\sum_{n \geq 1}(-t)^{n} \Gamma\left(e_{n}\right)}= \\
\frac{1}{1-\left(\left(z_{0}+\cdots+z_{k-1}\right) t+\sum_{n \geq 1} \frac{t^{n(j-1)+1}}{[n(j-1)+1]_{p, q} !}(x-1)^{n} m p_{(\tau, u), n(j-1)+1}^{k}\left(p, q, z_{0}, \ldots, z_{k-1}\right)\right)}
\end{gathered}
$$

which proves $(27)$.

If we replace $(\tau, u)$-matchings by exact $(\tau, u)$-matching throughout the proof of $(27)$, then we will get a proof of (28).

We note that in the special case of Theorem 6 where $k=1$, so that the underlying alphabet is is just $\{0\}$, a pair $\left(\tau, 0^{j}\right) \in C_{1} 2 S_{n}$ has the 1-minimal overlapping property if 
and only if $\tau$ has the minimal overlapping property. Thus if $k=0$ and we set $z_{0}=1$ in Theorem 6 , we obtain the following corollary.

Corollary 7. Suppose that $\tau \in S_{j}$ has the minimal overlapping property. Then

$$
\begin{aligned}
\sum_{n \geq 0} \frac{t^{n}}{[n]_{p, q} !} \sum_{\sigma \in S_{n}} x^{\tau-\operatorname{mch}(\sigma)} p^{\operatorname{coinv}(\sigma)} q^{\operatorname{inv}(\sigma)}= & 1 \\
& \frac{1}{1-\left(t+\sum_{n \geq 1} \frac{t^{n(j-1)+1}}{[n(j-1)+1]_{p, q} !}(x-1)^{n} m p_{\tau, n(j-1)+1}(p, q)\right)} .
\end{aligned}
$$

We end this section by observing that the proofs of Theorems 5 and 6 can be specialized to give another interesting pair of results. That is, we say that a word $u=u_{1} \ldots u_{j} \in$ $\{0, \ldots, k-1\}^{j}$ has the $k$-non-overlapping property ( $k$-exact match non-overlapping property) if the smallest $i$ such that there exists a word $w \in\{0, \ldots, k-1\}^{i}$ with $u$-mch $(w)=$ $2(E u-\operatorname{mch}(w)=2)$ is $2 j$. Thus $u$ has the $k$-non-overlapping ( $k$-exact match nonoverlapping) property if no two $u$-matches (exact $u$-matches) can share a letter. For example, if $k=3$ and $u=00112$, then it is easy to see that $u$ has both the $k$-nonoverlapping property and the $k$-exact match non-overlapping property. However, if $k=3$ and $v=011$, then $v$ has the $k$-exact match non-overlapping property but does not have the non-overlapping property since $w=01122$ has two $v$-matches. Similarly, we say that $(\tau, u) \in C_{k} 2 S_{j}$ has the $C_{k} 2 S_{n}$-non-overlapping property $\left(C_{k} 2 S_{n}\right.$-exact match nonoverlapping property) if the smallest $i$ such that there exists a $k$-colored permutation $(\sigma, w) \in C_{k} \zeta S_{i}$ with $(\tau, u)-\operatorname{mch}(w)=2((\tau, E u)-\operatorname{mch}(w)=2)$ is $2 j$. Clearly, there can be no permutations which have the non-overlapping property so that the only way a $(\tau, u) \in C_{k} \prec S_{j}$ can have the $C_{k} \prec S_{n}$-non-overlapping property $\left(C_{k} \prec S_{n}\right.$-exact match non-overlapping property) is if $u$ has the $k$-non-overlapping property ( $k$-exact match nonoverlapping property).

We can still define the notion of a maximum packing for words $u \in\{0, \ldots, k-1\}^{*}$ which have the $k$-non-overlapping ( $k$-exact match non-overlapping) property and for $k$ colored permutations which have the $C_{k} 2 S_{n}$-non-overlapping $\left(C_{k} 2 S_{n}\right.$-exact match nonoverlapping) property, it is just that in such cases the only maximum packings are of size $j$. It is then easy to check that the proofs of Theorems 5 and 6 go through exactly as before except that only Cases 1 and 2 of the involution apply. Thus we have the following theorems.

Theorem 8. Let $u \in\{0,1, \ldots, k-1\}^{j}$ where $j \geq 3$.

(I) If $u$ has the $k$-non-overlapping property and $\operatorname{red}(u)=u$, then

$$
\begin{aligned}
\sum_{n \geq 0} t^{n} \sum_{w \in\{0,1, \ldots, k-1\}^{n}} x^{u-\operatorname{mch}(w)} z(w)= & \frac{1}{1-\left(\left(z_{0}+\cdots+z_{k-1}\right) t+(x-1) m p_{u, j}^{k}\left(z_{0}, \ldots, z_{k-1}\right) t^{j}\right)} .
\end{aligned}
$$


(II) If $u$ has the $k$-exact match non-overlapping property, then

$$
\begin{aligned}
\sum_{n \geq 0} t^{n} \sum_{w \in\{0,1, \ldots, k-1\}^{n}} & x^{E u-\operatorname{mch}(w)} z(w)= \\
& \frac{1}{1-\left(\left(z_{0}+\cdots+z_{k-1}\right) t+(x-1) e m p_{u, j}^{k}\left(z_{0}, \ldots, z_{k-1}\right) t^{j}\right)}
\end{aligned}
$$

Theorem 9. (III) If $(\tau, u) \in C_{k} \imath S_{j}, \operatorname{red}(u)=u$, and $(\tau, u)$ has the $C_{k} \imath S_{n}$-nonoverlapping property, then

$$
\begin{aligned}
\sum_{n \geq 0} \frac{t^{n}}{n !} \sum_{(\sigma, w) \in C_{k} \imath S_{n}} x^{(\tau, u)-\operatorname{mch}((\sigma, u))} p^{\operatorname{coinv}(\sigma)} q^{\operatorname{inv}(\sigma)} z(w)= \\
\\
\frac{1}{1-\left(\left(z_{0}+\cdots+z_{k-1}\right) t+\frac{t^{j}}{[j]_{p, q} !}(x-1) m p_{(\tau, u), j}^{k}\left(p, q, z_{0}, \ldots, z_{k-1}\right)\right)}
\end{aligned}
$$

(IV) If $(\tau, u) \in C_{k} 2 S_{j}$ has the $C_{k} \imath S_{n}$-exact match non-overlapping property, then

$$
\begin{aligned}
\sum_{n \geq 0} \frac{t^{n}}{n !} \sum_{(\sigma, w) \in C_{k} l S_{n}} x^{(\tau, E u)-\operatorname{mch}((\sigma, u))} p^{\operatorname{coinv}(\sigma)} q^{\operatorname{inv}(\sigma)} z(w)= \\
\\
\frac{1}{1-\left(\left(z_{0}+\cdots+z_{k-1}\right) t+\frac{t^{j}}{[j]_{q} !}(x-1) e m p_{(\tau, u), j}^{k}\left(p, q, z_{0}, \ldots, z_{k-1}\right)\right)}
\end{aligned}
$$

\section{Computing the number of maximum packings}

In this section, we shall give several examples where we can compute the functions $m p_{u, n(j-1)+1}^{k}\left(z_{0}, \ldots, z_{k-1}\right), e m p_{u, n(j-1)+1}^{k}\left(z_{0}, \ldots, z_{k-1}\right), m p_{(\tau, u), n(j-1)+1}^{k}\left(p, q, z_{0}, \ldots, z_{k-1}\right)$, $e m p_{(\tau, u), n(j-1)+1}^{k}\left(p, q, z_{0}, \ldots, z_{k-1}\right)$, and $m p_{\tau, n(j-1)+1}(p, q)$.

First suppose that $u \in\{0,1, \ldots, k-1\}^{j}$ has the $k$-exact match minimal overlapping property. Then it is trivial to compute $e m p_{u, n(j-1)+1}^{k}\left(z_{0}, \ldots, z_{k-1}\right)$. That is, if $u=u_{1} \ldots u_{j}$, then we must have $u_{1}=u_{j}$ so that it is simple to see that $e m p_{u, n(j-1)+1}^{k}\left(z_{0}, \ldots, z_{k-1}\right)=$ $z_{u_{1}}\left(z_{u_{2}} \cdots z_{u_{j}}\right)^{n}$. Thus for example, if $u=01^{a} 0$ where $a \geq 1$, then

$$
e m p_{01^{a} 0, n(j-1)+1}^{k}\left(z_{0}, \ldots, z_{k-1}\right)=z_{0}\left(z_{0} z_{1}^{a}\right)^{n}=z_{0}^{n+1} z_{1}^{a n}
$$

for all $k \geq 2$.

Next suppose that $u=u_{1} \ldots u_{j} \in\{0,1, \ldots, k-1\}^{j}$ has the $k$-minimal overlapping property and $\operatorname{red}(u)=u$. In the case where $u_{1}=u_{j}$, it is again relatively straightforward to compute $m p_{u, n(j-1)+1}^{k}\left(z_{0}, \ldots, z_{k-1}\right)$. For example if $u=01^{a} 0$ where $a \geq 1$, then all maximum packings for $u$ of length $n(a+1)+1$ are of the form

$$
i b_{1}^{a} i b_{2}^{a} i \ldots i b_{n}^{a} i
$$

THE ELECTRONiC JOURNAL OF COMBinAtorics 18 (2011), \#P25 
where $b_{1}, \ldots, b_{n} \in\{i+1, \ldots, k-1\}$. Thus

$$
m p_{01^{a} 0,2 n+1}^{k}\left(z_{0}, \ldots, z_{k-1}\right)=\sum_{i=0}^{k-2} z_{i}^{n+1}\left(\sum_{j=i+1}^{k-1} z_{j}^{a}\right)^{n} .
$$

If $v=10^{a} 1$ where $a \geq 1$, then all maximum packings for $v$ of length $n(a+1)+1$ are of the form

$$
i b_{1}^{a} i b_{2}^{a} i \ldots i b_{n}^{a} i
$$

where $b_{1}, \ldots, b_{n} \in\{0, \ldots, i-1\}$. Thus

$$
m p_{10^{a} 1,2 n+1}^{k}\left(z_{0}, \ldots, z_{k-1}\right)=\sum_{i=1}^{k-1} z_{i}^{n+1}\left(\sum_{j=0}^{i-1} z_{j}^{a}\right)^{n} .
$$

However, it is no longer the case that we are forced to have $u_{1}=u_{j}$ if $u$ has the $k$-minimal overlapping property. For example if $k \geq 3$ and $u=001$, then $w=00112$ has $2 u$-matches so the 001 has the $k$-minimal overlapping property for $k \geq 3$. We note that in such cases, there cannot be maximal packings of arbitrary length. That is, suppose that $u_{1}<u_{j}$. Then it is easy to see that in a maximum packing $w=w_{1} \ldots w_{n(j-1)+1}$ for $u$, we must have that

$$
w_{1}<w_{j}<w_{2(j-1)+1}<w_{3(j-1)+1}<\cdots<w_{n(j-1)+1}
$$

so that it is impossible to have $n \geq k$. A similar argument applies in the case where $u_{1}>$ $u_{j}$. It follows from Theorem 5 that if $u=u_{1} \ldots u_{j} \in\{0,1, \ldots, k-1\}^{j}$ has the $k$-minimal overlapping property and $u_{1} \neq u_{j}$, then $\sum_{n \geq 0} t^{n} \sum_{w \in\{0,1, \ldots, k-1\}^{n}} x^{u-\operatorname{mch}(w)} z(w)$ is a rational function. In general, it is still rather straightforward to compute $m p_{u, n(j-1)+1}^{k}\left(z_{0}, \ldots, z_{k-1}\right)$. For example, if $u=001$, then all maximum packings for $u$ must be of the form

$$
a_{1} a_{1} a_{2} a_{2} \ldots a_{r} a_{r} a_{r+1}
$$

where $0 \leq a_{1}<\cdots<a_{r+1} \leq k-1$. Thus

$$
m p_{001,2 n+1}^{k}\left(z_{0}, \ldots, z_{k-1}\right)= \begin{cases}0 & \text { if } n>k \\ z_{k-1} \prod_{i=0}^{k-2} z_{i}^{2} & \text { if } n=k \\ \sum_{0 \leq a_{1}<\cdots<a_{n+1} \leq k-1} z_{a_{n+1}} \prod_{i=1}^{n} z_{a_{i}}^{2} & \text { if } 1 \leq n<k .\end{cases}
$$

If $\tau \in S_{j}$ has the minimal overlapping property, then it seems to be a difficult problem to compute $m p_{\tau, n(j-1)+1}(p, q)$ in general. However, there is at least one special case where it is easy, namely, when $\tau$ starts with 1 . That is, in that case, we have the following theorem.

Theorem 10. Suppose that $\tau=\tau_{1} \ldots \tau_{j}$ has the minimal overlapping property where $\tau_{1}=1$ and $\tau_{j}=s$. Then for all $n \geq 1$.

$$
m p_{\tau,(n+1)(j-1)+1}(p, q)=
$$




$$
p^{\operatorname{coinv}(\tau)} q^{i n v(\tau)} p^{(s-1) n(j-1)}\left[\begin{array}{c}
(n+1)(j-1)+1-s \\
j-s
\end{array}\right]_{p, q} m p_{\tau, n(j-1)+1}(p, q)
$$

so that

$$
m p_{\tau,(n+1)(j-1)+1}(p, q)=\left(p^{\operatorname{coinv}(\tau)} q^{i n v(\tau)}\right)^{(n+1)} p^{(s-1)(j-1)\left(\begin{array}{c}
n+1 \\
2
\end{array}\right)} \prod_{i=1}^{n+1}\left[\begin{array}{c}
i(j-1)+1-s \\
j-s
\end{array}\right]_{p, q} .
$$

Proof. Suppose that $\sigma=\sigma_{1} \ldots \sigma_{(n+1)(j-1)+1}$ is a maximum packing for $\tau$. Since $\tau_{1}=1$, it must be the case that $\sigma_{1}$ is less than $\sigma_{2}, \ldots, \sigma_{j}, \sigma_{j}$ is less than $\sigma_{j+1}, \ldots, \sigma_{j+(j-1)}, \sigma_{2 j-1}$ is less than $\sigma_{2 j}, \ldots, \sigma_{2 j-1+(j-1)}$, etc.. It follows that $\sigma_{1}=1$ and that $\sigma_{j}$ must be less than $\sigma_{j+1}, \ldots, \sigma_{(n+1)(j-1)+1}$. We claim that $\sigma_{j}$ must be $s$. We know that $\sigma_{j} \geq s$ because the fact that $\sigma_{1} \ldots \sigma_{j}$ is a $\tau$-match means that there must be $s-1$ elements of $\sigma_{1}, \ldots, \sigma_{j-1}$ which are less than $\sigma_{j}$. However if $\sigma_{j}>s$, then $1, \ldots, \sigma_{j}-1$ must be among $\sigma_{1}, \ldots, \sigma_{j-1}$ which would violate the fact that $\sigma_{j}$ is the $s$-th smallest element among $\sigma_{1}, \ldots, \sigma_{j}$.

It follows that $1, \ldots, s$ must be among $\sigma_{1}, \ldots, \sigma_{j}$ which means that the positions of $1, \ldots, s$ in $\sigma_{1} \ldots \sigma_{j}$ must be the same as the positions of $1, \ldots, s$ in $\tau$. But then we have

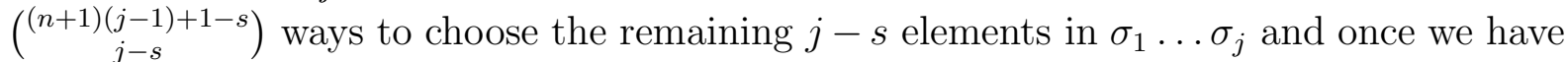
chosen these $j-s$ elements, their positions are completely determined by $\tau$ since $\sigma_{1} \ldots \sigma_{j}$ is a $\tau$-match. Moreover $\operatorname{pred}\left(\sigma_{j} \ldots \sigma_{(n+1)(j-1)+1}\right)$ must be an element of $\mathcal{M} \mathcal{P}_{\tau, n(j-1)+1}$. It then follows that

$$
m p_{\tau,(n+1)(j-1)+1}=\left(\begin{array}{c}
(n+1)(j-1)+1-s \\
j-s
\end{array}\right) m p_{\tau, n(j-1)+1} .
$$

Next consider the problem of computing $m p_{\tau,(n+1)(j-1)+1}(p, q)$. For any $\sigma$ in $\mathcal{M P}_{\tau,(n+1)(j-1)+1}$, we can organize the count of inversions and coinversions of $\sigma$ by

(a) counting the inversions and coinversions among $\sigma_{1} \ldots \sigma_{j}$ which contributes a factor of $p^{\operatorname{coinv}(\tau)} q^{\operatorname{inv}(\tau)}$ to $p^{\operatorname{coinv}(\sigma)} q^{\operatorname{inv}(\sigma)}$,

(b) counting the inversions and coinversions between $1, \ldots s-1$ and $\sigma_{j+1} \ldots \sigma_{(n+1(j-1)+1}$ which contributes a factor of $p^{(s-1) n(j-1)}$ to $p^{\operatorname{coinv}(\sigma)} q^{\operatorname{inv}(\sigma)}$,

(c) counting the inversions and coinversions among $\left\{\sigma_{1}, \ldots, \sigma_{j}\right\}-\{1, \ldots, s\}$ and $\sigma_{j+1} \ldots \sigma_{(n+1)(j-1)+1}$ which contributes a factor of $\left[\begin{array}{c}(n+1)(j-1)+1-s \\ j-s\end{array}\right]_{p, q}$ to $m p_{\tau,(n+1)(j-1)+1}(p, q)$ as we vary over all choices $\left\{\sigma_{1}, \ldots, \sigma_{j}\right\}-\{1, \ldots, s\}$, and

(d) counting the inversions and coinversions among $\sigma_{j} \ldots \sigma_{(n+1)(j-1)+1}$.

Thus

$$
\begin{aligned}
& m p_{\tau,(n+1)(j-1)+1}(p, q)= \\
& p^{\operatorname{coinv}(\tau)} q^{i n v(\tau)} p^{(s-1) n(j-1)}\left[\begin{array}{c}
(n+1)(j-1)+1-s \\
j-s
\end{array}\right]_{p, q} m p_{\tau, n(j-1)+1}(p, q) .
\end{aligned}
$$

Iterating this recursion then yields (38). 
By our remarks in the introduction, if $\tau \in S_{j}$ has the minimal overlapping property, then $\tau^{r}$ and $\tau^{c}$ have the minimal overlapping property. Thus we can use Theorem 10 to find formulas for $m p_{\tau,(n+1)(j-1)+1}(p, q)$ when either $\tau$ starts or ends in $j$ or ends in 1 .

It follows from Corollary 7 and Theorem 10 that if $j \geq 3$ and $\tau=\tau_{1} \ldots \tau_{j} \in S_{j}$ has the minimal overlapping property, $\tau_{1}=1$ and $\tau_{j}=s$, then

$$
\begin{aligned}
& P_{\tau}(t, x, p, q)=\sum_{n \geq 0} \frac{t^{n}}{[n]_{p, q} !} \sum_{\sigma \in S_{n}} x^{\tau-\operatorname{mch}(\sigma)} p^{\operatorname{coinv}(\sigma)} q^{\operatorname{inv}(\sigma)}= \\
& \frac{1}{1-\left(t+\sum_{n \geq 1} \frac{(x-1)^{n} t^{n(j-1)+1}}{[n(j-1)+1]_{p, q} !}\left(p^{\operatorname{coinv}(\tau)} q^{\operatorname{inv}(\tau)}\right)^{(n+1)} p^{(s-1)(j-1)\left(\begin{array}{c}
n+1 \\
2
\end{array}\right)} \prod_{i=1}^{n+1}\left[\begin{array}{c}
i(j-1)+1-s \\
j-s
\end{array}\right]_{p, q}\right)} .
\end{aligned}
$$

For example, both 132 and 1342 have the minimal overlapping property. As stated in the introduction, Elizalde and Noy [8] proved that

$$
\begin{aligned}
P_{132}(t, q) & =\sum_{n \geq 0} \frac{t^{n}}{n !} \sum_{\sigma \in S_{n}} x^{132-\operatorname{mch}(\sigma)} \\
& =\frac{1}{1-\int_{0}^{t} e^{(x-1) z^{2} / 2} d z} \\
& =\frac{1}{1-\sum_{n \geq 0} \frac{(x-1)^{n} t^{2 n+1}}{2^{n}(n !)(2 n+1)}} .
\end{aligned}
$$

By Theorem 10, we have

$$
m p_{132,2 n+1}(p, q)=\left(p^{2} q\right)^{n} p^{2\left(\begin{array}{c}
n \\
2
\end{array}\right)} \prod_{i=1}^{n}[2 i-1]_{p, q} .
$$

Plugging equation (40) into Corollary 7, we get the following $p, q$-analogue of Elizalde and Noy's result for $P_{132}(t, x)$.

$$
\begin{aligned}
P_{132}(t, x, p, q) & =\sum_{n \geq 0} \frac{t^{n}}{[n]_{p, q} !} \sum_{\sigma \in S_{n}} x^{132-\operatorname{mch}(\sigma)} p^{\operatorname{coinv}(\sigma)} q^{\operatorname{inv}(\sigma)} \\
& =\frac{1}{1-\left(t+\sum_{n \geq 1} \frac{(x-1)^{n} t^{2 n+1}}{[2 n+1]_{p, q} !} p^{n^{2}+n} q^{n} \prod_{i=1}^{n}[2 i-1]_{p, q}\right)} \\
& =\frac{1}{1-\sum_{n \geq 0} \frac{p^{n^{2}+n} q^{n}(x-1)^{n} t^{2 n+1}}{[2 n+1]_{p, q} \prod_{i=1}^{n}[2]_{p, q}}} .
\end{aligned}
$$

Similarly, Elizalde and Noy [8] proved that

$$
P_{1342}(t, x)=\sum_{n \geq 0} \frac{t^{n}}{n !} \sum_{\sigma \in S_{n}} x^{1342-\operatorname{mch}(\sigma)}
$$




$$
\begin{aligned}
& =\frac{1}{1-\int_{0}^{t} e^{(x-1) z^{3} / 6} d z} \\
& =\frac{1}{1-\sum_{n \geq 0} \frac{(x-1)^{n} t^{3 n+1}}{6^{n}(n !)(3 n+1)}}
\end{aligned}
$$

By Theorem 10, we have

$$
\begin{aligned}
m p_{1342,2 n+1} & =\left(p^{3} q^{2}\right)^{n} p^{3\left(\begin{array}{l}
n \\
2
\end{array}\right)} \prod_{i=1}^{n}\left[\begin{array}{c}
3 n+1-2 \\
2
\end{array}\right]_{p, q} \\
& =p^{\left(3 n^{2}+3 n\right) / 2} q^{2 n} \prod_{i=1}^{n} \frac{[3 n-1]_{p, q}[3 n-2]_{p, q}}{[2]_{p, q}} .
\end{aligned}
$$

Plugging equation (41) into Corollary 7, we get the following $p, q$-analogue of Elizalde and Noy's result for $P_{1342}(t, x)$.

$$
\begin{aligned}
P_{1342}(t, x, p, q) & =\sum_{n \geq 0} \frac{t^{n}}{[n]_{p, q} !} \sum_{\sigma \in S_{n}} x^{1342-\operatorname{mch}(\sigma)} p^{\operatorname{coinv}(\sigma)} q^{\operatorname{inv}(\sigma)} \\
& =\frac{1}{1-\left(t+\sum_{n \geq 1} \frac{(x-1)^{n} t^{3 n+1}}{[3 n+1]_{p, q} !} p^{\left(3 n^{2}+3 n\right) / 2} q^{2 n} \frac{1}{[2]_{p, q}^{n}} \prod_{i=1}^{n}[3 i-1]_{p, q}[3 i-2]_{p, q}\right)} \\
& =\frac{1}{1-\sum_{n \geq 0} \frac{p^{\left(3 n^{2}+3 n\right) / 2} q^{2 n}(x-1)^{n} t^{3 n+1}}{[3 n+1]_{p, q}[2]_{p, q}^{n} \prod_{i=1}^{n}[3 i]_{p, q}}} .
\end{aligned}
$$

Now if $\tau=12 \ldots a \sigma a+1$ where $\sigma$ is a permutation of $\{a+2, \ldots, k+1\}$, then $\tau$ has the minimal overlapping property. Note that

$$
\begin{aligned}
\operatorname{coinv}(\tau) & =\left(\begin{array}{c}
a+1 \\
2
\end{array}\right)+a(k-a)+\operatorname{coinv}(\sigma) \text { and } \\
\operatorname{inv}(\tau) & =(k-a)+\operatorname{inv}(\sigma) .
\end{aligned}
$$

Thus using Corollary 7 and Theorem 10, we can derive the following generalization of Kitaev's Theorem 2.

Theorem 11. Let $\tau=12 \ldots a \sigma a+1$ where $\sigma$ is a permutation of $\{a+2, \ldots, k+1\}$. Then

$$
\begin{aligned}
& \sum_{n \geq 0} \frac{t^{n}}{[n]_{p, q} !} \sum_{\sigma \in S_{n}} x^{\tau-\operatorname{mch}(\sigma)} p^{\operatorname{coinv}(\sigma)} q^{\operatorname{inv}(\sigma)}= \\
& \frac{1}{1-\left(t+\sum_{i \geq 0} \frac{(x-1)^{i+1} t^{i k+1}}{[i k+1]_{p, q} !}\left(p^{a(k-a)+\operatorname{coinv}(\sigma)+\left(\begin{array}{c}
a+1 \\
2
\end{array}\right)} q^{k-a+\operatorname{inv}(\sigma)}\right)^{(i+1)} p^{a k\left(\begin{array}{c}
i+1 \\
2
\end{array}\right)} \prod_{j=2}^{i}\left[\begin{array}{c}
j k-a \\
k-a
\end{array}\right]_{p, q}\right.} .
\end{aligned}
$$

Next following a suggestion of Sergey Kitaev, we show that if $\alpha$ and $\beta$ are two permutations in $S_{j}$ which have the minimal overlapping property and have the same first and last elements, then for any $n, m p_{\alpha, n(j-1)+1}=m p_{\beta, n(j-1)+1}$. That is, we shall prove the following. 
Theorem 12. Suppose $\alpha=\alpha_{1} \ldots \alpha_{j}$ and $\beta=\beta_{1} \ldots \beta_{j}$ are minimal overlapping permutations in $S_{j}$ and $\alpha_{1}=\beta_{1}$ and $\alpha_{j}=\beta_{j}$, then for all $n \geq 1$,

$$
m p_{\alpha, n(j-1)+1}=m p_{\beta, n(j-1)+1} .
$$

If in addition, $p^{\operatorname{coinv}(\alpha)} q^{\operatorname{inv}(\alpha)}=p^{\operatorname{coinv}(\beta)} q^{\operatorname{inv}(\beta)}$, then

$$
m p_{\alpha, n(j-1)+1}(p, q)=m p_{\beta, n(j-1)+1}(p, q) .
$$

Proof. Suppose that we wanted to construct all maximum packings $\sigma=\sigma_{1} \ldots \sigma_{n(j-1)+1}$ of size $n(j-1)+1$ for $\alpha$ or $\beta$. One way to do this is to partition $\{1, \ldots, n(j-1)+1\}$ into sets $T_{1}, \ldots, T_{n}$ where $\left|T_{1}\right|=j$ and $\left|T_{i}\right|=j-1$ for $i \geq 2$ and use the elements of $T_{1}$ for $\sigma_{1} \ldots \sigma_{j}$, use the elements of $T_{2}$ for $\sigma_{j+1} \ldots \sigma_{2 j-1}$, use the elements of $T_{3}$ for $\sigma_{2 j} \ldots \sigma_{3 j-2}$, etc.. Of course, this will not work for all choices of $T_{1}, \ldots T_{n}$. That is, if $\alpha=132$ and we pick $T_{1}=\{4,5,6\}$ and $T_{2}=\{1,2\}$, then there will be no way to use $T_{1}$ for the elements $\sigma_{1} \sigma_{2} \sigma_{3}$ and use $T_{2}$ for the elements $\sigma_{4} \sigma_{5}$ to produce a maximum packing for 132. That is, in such a situation, we must let $\sigma_{1}=3, \sigma_{2}=5$, and $\sigma_{3}=4$. But then $\sigma_{3}$ will be greater than $\sigma_{4}$ and $\sigma_{5}$ so that this choice will not allow us to construct a maximum packing for 132 . Our claim is that for any choice of $T_{1}, \ldots, T_{n}$, either we cannot construct a maximum packing for either $\alpha$ or $\beta$ in this way or we can construct a maximum packing for both $\alpha$ and $\beta$ in this way. For example, if $\alpha=24153$, then we have pictured in Figure 7 choices for $T_{1}, T_{2}, T_{3}$, and $T_{4}$ and the steps needed to construct a maximum packing for $\alpha$. Clearly, given $T_{1}$, there is only one way to place the elements of $T_{1}$ so that $\sigma_{1} \ldots \sigma_{5}$ is a $\alpha$-match. This is pictured in the second row of Figure 7 . In this process, $\sigma_{5}=6$ must be the third largest element of $T_{1}$ since $\alpha_{5}=3$. But then to continue, it must be the case that 6 is the second largest element of $\{6\} \cup T_{2}$ if $\sigma_{5} \ldots \sigma_{9}$ is an $\alpha$-match. Since 6 is the second largest element in $\{6\} \cup T_{2}$, the positions of the elements of $T_{2}$ are then forced by the requirement that $\sigma_{5} \ldots \sigma_{9}$ be an $\alpha$-match which is pictured in row three of Figure 7 . In particular, $\sigma_{9}$ must be the third largest element of $\{6\} \cup T_{2}$ so that $\sigma_{9}=8$. To continue, it must be the case that 8 is the second largest element of $\{8\} \cup T_{3}$ if $\sigma_{9} \ldots \sigma_{13}$ is an $\alpha$-match and $\sigma_{13}$ must be the third largest element of $\{8\} \cup T_{3}$. In this case, 8 is the second largest element of $\{8\} \cup T_{3}$ and we are forced to have $\sigma_{13}=11$ since 11 is the third largest element of $\{8\} \cup T_{3}$. Finally, it must be the case that 11 is the second largest element of $\{11\} \cup T_{4}$ which is true in this case so that we can complete the construction of a maximum packing for $\alpha$ using $T_{1}, \ldots, T_{4}$.

In general, suppose that $\alpha_{1}=\beta_{1}=s$ and $\alpha_{j}=\beta_{j}=t$, Then to be able to use $T_{2}$ to continue the construction of a maximum packing for either $\alpha$ or $\beta$, we must have that the $t$-th largest element $a_{1}$ of $T_{1}$ is the $s$-th largest element of $\left\{a_{1}\right\} \cup T_{2}$. If not, we cannot use $T_{1}, \ldots, T_{n}$ to construct a maximum packing for either $\alpha$ or $\beta$. If so, then the $t$-th largest element $a_{2}$ of $\left\{a_{1}\right\} \cup T_{2}$ must be the $s$-largest element of $\left\{a_{2}\right\} \cup T_{3}$. If not, we cannot use $T_{1}, \ldots, T_{n}$ to construct a maximum packing for either $\alpha$ or $\beta$. If so, then the $t$-th largest element $a_{3}$ of $\left\{a_{2}\right\} \cup T_{3}$ must be the $s$-largest element of $\left\{a_{3}\right\} \cup T_{4}$, etc. Thus it is easy to see that we can use $T_{1}, \ldots, T_{n}$ to construct a maximum packing $\sigma$ for $\alpha$ if and only if we can use $T_{1}, \ldots, T_{n}$ to construct a maximum packing $\sigma^{*}$ for $\beta$. 


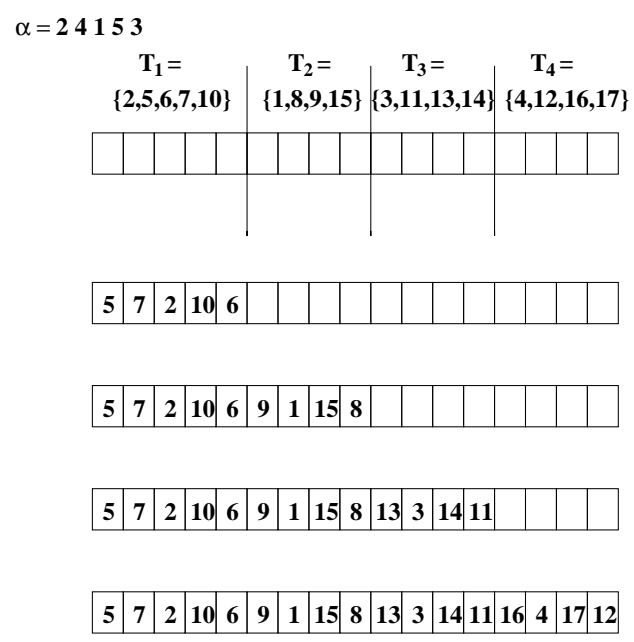

Figure 7: Constructing maximum packings for $\alpha=24153$.

Moreover, it is easy to see that if $p^{\operatorname{coinv}(\alpha)} q^{\operatorname{inv}(\alpha)}=p^{\operatorname{coinv}(\beta)} q^{\operatorname{inv}(\beta)}$, then it will be the case that $p^{\operatorname{coinv}(\sigma)} q^{\operatorname{inv}(\sigma)}=p^{\operatorname{coinv}\left(\sigma^{*}\right)} q^{\operatorname{inv}\left(\sigma^{*}\right)}$. Thus (43) and (44) hold.

We can now use Theorem 12 and Corollary 7 to prove Elizalde's conjecture stated in the introduction. That is, suppose that $\alpha=\alpha_{1} \ldots \alpha_{j}$ and $\beta=\beta_{1} \ldots \beta_{j}$ are minimal overlapping permutations in $S_{j}$ and $\alpha_{1}=\beta_{1}$ and $\alpha_{j}=\beta_{j}$. Elizalde [7] conjectured that it must be the case that $\alpha$ and $\beta$ are strongly c-Wilf equivalent. By Theorem 12, we know that in such a situation, $m p_{\alpha, n(j-1)+1}=m p_{\beta, n(j-1)+1}$ for all $n \geq 1$ and, hence, by Corollary 7 with $p=q=1$, we know that

$$
\begin{aligned}
& \sum_{n \geq 0} \frac{t^{n}}{n !} \sum_{\sigma \in S_{n}} x^{\alpha-\operatorname{mch}(\sigma)}= \\
& \frac{1}{1-\left(t+\sum_{n \geq 1} \frac{t^{n(j-1)+1}}{(n(j-1)+1) !}(x-1)^{n} m p_{\alpha, n(j-1)+1}\right)}= \\
& \frac{1}{1-\left(t+\sum_{n \geq 1} \frac{t^{n(j-1)+1}}{(n(j-1)+1) !}(x-1)^{n} m p_{\beta, n(j-1)+1}\right)}= \\
& \sum_{n \geq 0} \frac{t^{n}}{n !} \sum_{\sigma \in S_{n}} x^{\beta-\operatorname{mch}(\sigma)} .
\end{aligned}
$$

Thus Elizalde's conjecture follows. If in addition, $p^{\operatorname{coinv}(\alpha)} q^{\operatorname{inv}(\alpha)}=p^{\operatorname{coinv}(\beta)} q^{\operatorname{inv}(\beta)}$, then by the same reasoning, we get the even stronger conclusion that

$$
\sum_{n \geq 0} \frac{t^{n}}{[n]_{p, q} !} \sum_{\sigma \in S_{n}} x^{\alpha-\operatorname{mch}(\sigma)} p^{\operatorname{coinv}(\alpha)} q^{\operatorname{inv}(\alpha)}=\sum_{n \geq 0} \frac{t^{n}}{[n]_{p, q} !} \sum_{\sigma \in S_{n}} x^{\beta-\operatorname{mch}(\sigma)} p^{\operatorname{coinv}(\beta)} q^{\operatorname{inv}(\beta)} .
$$

We note that Elizalde's conjecture has been proved independently by Vladimir Dotsenko and Anton Khoroshkin [4] using cluster algebras and ideas similar to Theorem 12 . 
Next we consider the problem of computing $m p_{(\tau, u), n(j-1)+1}^{k}\left(p, q, z_{0}, \ldots, z_{k-1}\right)$ $\left(e m p_{(\tau, u), n(j-1)+1}^{k}\left(p, q, z_{0}, \ldots, z_{k-1}\right)\right)$ for $(\tau, u) \in C_{k}$ 々 $S_{n}$ which have the $C_{k}$ 々 $S_{n}$-minimal overlapping property $\left(C_{k}<S_{n}\right.$-exact match minimal overlapping property). There are several reasons why a $(\tau, u) \in C_{k} \imath S_{j}$ can have the $C_{k} \imath S_{n}$-minimal overlapping property. For example, it could be that $u$ has the $k$-minimal overlapping property such as $(\tau, u)=$ $\left(12 \ldots a+2,01^{a} 0\right)$. In this case it is easy to see that

$$
\left.m p_{(\tau, u), n(j-1)+1}^{k}\left(p, q, z_{0}, \ldots, z_{k-1}\right)=p_{2}^{(n(j-1)+1}\right) m p_{u, n(j-1)+1}^{k}\left(z_{0}, \ldots, z_{k}\right) .
$$

It could also be that $\tau$ has the minimal overlapping property such as $(\tau, u)=(132,000)$. In this case, it is easy to see that

$$
m p_{(\tau, u), n(j-1)+1}^{k}\left(p, q, z_{0}, \ldots, z_{k-1}\right)=m p_{\tau, n(j-1)+1}^{k}(p, q) \sum_{i=0}^{k-1} z_{i}^{n(j-1)+1} .
$$

If it is the case that $u$ has the $k$-minimal overlapping property and $\tau$ has the minimal overlapping property, then it is easy to see that

$$
m p_{(\tau, u), n(j-1)+1}^{k}\left(p, q, z_{0}, \ldots, z_{k-1}\right)=m p_{\tau, n(j-1)+1}(p, q) m p_{u, n(j-1)+1}^{k}\left(z_{0}, \ldots, z_{k}\right) .
$$

However, there are cases like $(\tau, u)=(152364,001100)$ which has the $C_{k} \zeta S_{n}$-minimal overlapping property where neither $u$ has the $k$-minimal overlapping property nor $\tau$ has the minimal overlapping property. Now if $\tau$ starts with 1 , then we can mimic the reasoning in Theorem 10 to find an explicit formula for $m p_{(\tau, u), n(j-1)+1}^{k}\left(p, q, z_{0}, \ldots, z_{k-1}\right)$. That is, suppose $\tau=\tau_{1} \ldots \tau_{j} \in S_{j}$ is a permutation such that $\tau_{1}=1$ and $\tau_{j}=s$. Then let $M P_{\tau, n(j-1)+1}^{\{1, j, 2 j-1, \ldots,(n-1) j-(n-2)\}}$ denote the set of permutations in $S_{n(j-1)+1}$ which have $\tau$ matches starting at positions $1, j, 2 j-1,3 j-2, \ldots,(n-1) j-(n-2)$ and let

$$
m p_{\tau, n(j-1)+1}^{\{1, j, 2 j-1, \ldots,(n-1) j-(n-2)\}}(p, q)=\sum_{\sigma \in M P_{\tau, n(j-1)+1}^{\{1, j, 2 j-1, \ldots,(n-1) j-(n-2)\}}} p^{\operatorname{coinv}(\sigma)} q^{\operatorname{inv}(\sigma)} .
$$

If $u=u_{1} \ldots u_{j} \in\{0,1, \ldots, k-1\}^{j}$ and $\operatorname{red}(u)=u$, we let $M P_{u, n(j-1)+1}^{k,\{1, j, 2 j-1, \ldots,(n-1) j-(n-2)\}}$ denote the set of all words $w \in\{0,1, \ldots, k-1\}^{n(j-1)+1}$ which have $u$-matches starting at positions $1, j, 2 j-1,3 j-2, \ldots,(n-1) j-(n-2)$ and let

$$
m p_{u, n(j-1)+1}^{k,\{1, j, 2 j-1, \ldots,(n-1) j-(n-2)\}}\left(z_{0}, \ldots, z_{k-1}\right)=\sum_{w \in M P_{u, n(j-1)+1}^{k,\{1, j, 2 j-1, \ldots,(n-1) j-(n-2)\}}} z(w) .
$$

Similarly, if $u=u_{1} \ldots u_{j} \in\{0,1, \ldots, k-1\}^{j}$, we let $E M P_{u, n(j-1)+1}^{k,\{1, j, 2 j-1, \ldots,(n-1) j-(n-2)\}}$ denote the set of all words $w \in\{0,1, \ldots, k-1\}^{n(j-1)+1}$ which have exact $u$-matches starting at positions $1, j, 2 j-1,3 j-2, \ldots,(n-1) j-(n-2)$ and let

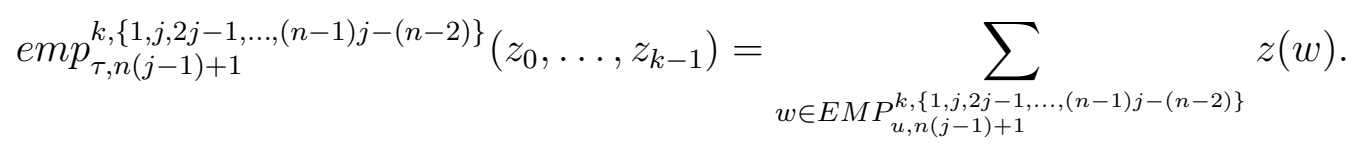


Then using the same reasoning as in Theorem 10, we can show that

$$
\begin{aligned}
& m p_{\tau,(n+1)(j-1)+1}^{\{1, j, 2 j-1, \ldots,(n-1) j-(n-2), n j-(n-1)\}}(p, q)= \\
& p^{\operatorname{coinv}(\tau)} q^{\operatorname{inv}(\tau)} p^{(s-1) n(j-1)}\left[\begin{array}{c}
(n+1)(j-1)+1-s \\
j-s
\end{array}\right]_{p, q} m p_{\tau, n(j-1)+1}^{\{1, j, 2 j-1, \ldots,(n-1) j-(n-2)\}}(p, q)
\end{aligned}
$$

so that

$$
\begin{aligned}
& m p_{\tau,(n+1)(j-1)+1}^{\{1, j, 2 j-1, \ldots,(n-1) j-(n-2)\}}\left(p, q, z_{0}, \ldots, z_{k-1}\right)= \\
& \left(p^{\operatorname{coinv}(\tau)} q^{i n v(\tau)}\right)^{(n+1)} p^{(s-1)(j-1)\left(\begin{array}{c}
n+1 \\
2
\end{array}\right)} \prod_{i=1}^{n+1}\left[\begin{array}{c}
i(j-1)+1-s \\
j-s
\end{array}\right]_{p, q} .
\end{aligned}
$$

We then have the following theorem.

Theorem 13. Suppose that $(\tau, u) \in C_{k} 2 S_{n}$ has the $C_{k} 2 S_{n}$-minimal overlapping property, $\operatorname{red}(u)=u$, and $\tau=\tau_{1} \ldots \tau_{j}$ where $\tau_{1}=1$ and $\tau_{j}=s$. Then

$$
\begin{aligned}
& m p_{(\tau, u), n(j-1)+1}^{k}\left(p, q, z_{0}, \ldots, z_{k-1}\right)= \\
& m p_{\tau, n(j-1)+1}^{\{1, j, 2 j-1, \ldots,(n-1) j-(n-2)\}}(p, q) m p_{u, n(j-1)+1}^{k,\{1, j, 2 j-1, \ldots,(n-1) j-(n-2)\}}\left(z_{0}, \ldots, z_{k}\right)= \\
& m p_{u, n(j-1)+1}^{k,\{1, j, 2 j-1, \ldots,(n-1) j-(n-2)\}}\left(z_{0}, \ldots, z_{k}\right) \times \\
& \left(p^{\operatorname{coinv}(\tau)} q^{i n v(\tau)}\right)^{n} p^{(s-1)(j-1)\left(\begin{array}{l}
n \\
2
\end{array}\right)} \prod_{i=1}^{n}\left[\begin{array}{c}
i(j-1)+1-s \\
j-s
\end{array}\right]_{p, q} .
\end{aligned}
$$

Similarly if $(\tau, u) \in C_{k} 2 S_{n}$ has the $C_{k} 2 S_{n}$-exact match minimal overlapping property and $\tau=\tau_{1} \ldots \tau_{j}$ where $\tau_{1}=1$ and $\tau_{j}=s$, then

$$
\begin{aligned}
& e m p_{(\tau, u), n(j-1)+1}^{k}\left(p, q, z_{0}, \ldots, z_{k-1}\right)= \\
& m p_{\tau, n(j-1)+1}^{\{1,2 j-1, \ldots,(n-1) j-(n-2)\}}(p, q) e m p_{u, n(j-1)+1}^{k,\{1, j, 2 j-1, \ldots,(n-1) j-(n-2)\}}\left(z_{0}, \ldots, z_{k}\right)= \\
& e m p_{u, n(j-1)+1}^{k,\{1, j, 2 j-1, \ldots,(n-1) j-(n-2)\}}\left(z_{0}, \ldots, z_{k}\right) \times \\
& \left(p^{\operatorname{coinv}(\tau)} q^{i n v(\tau)}\right)^{n} p^{(s-1)(j-1)\left(\begin{array}{l}
n \\
2
\end{array}\right)} \prod_{i=1}^{n}\left[\begin{array}{c}
i(j-1)+1-s \\
j-s
\end{array}\right]_{p, q} .
\end{aligned}
$$

Now suppose that $\alpha=\alpha_{1} \ldots \alpha_{j}$ and $\beta=\beta_{1} \ldots \beta_{j}$ are two permutations in $S_{j}$ such that $\alpha_{1}=\beta_{1}$ and $\alpha_{j}=\beta_{j}$ and $u \in\{0, \ldots, k-1\}^{j}$ is such that $(\alpha, u)$ and $(\beta, u)$ have the $C_{k} 2 S_{n}$-minimal overlapping property $\left(C_{k} 2 S_{n}\right.$-exact match minimal overlapping property). Then we can use the same reasoning at Theorem 12 to show that for all $n \geq 1$,

$$
m p_{\alpha, n(j-1)+1}^{\{1, j, 2 j-1, \ldots,(n-1) j-(n-2)\}}=m p_{\beta, n(j-1)+1}^{\{1, j, 2 j-1, \ldots,(n-1) j-(n-2)\}} .
$$

If, in addition, $p^{\operatorname{coinv}(\alpha)} q^{\operatorname{inv}(\alpha)}=p^{\operatorname{coinv}(\beta)} q^{\operatorname{inv}(\beta)}$, then

$$
m p_{\alpha, n(j-1)+1}^{\{1, j, 2 j-1, \ldots,(n-1) j-(n-2)\}}(p, q)=m p_{\beta, n(j-1)+1}^{\{1, j, 2 j-1, \ldots,(n-1) j-(n-2)\}}(p, q) .
$$


Since for $\tau \in\{\alpha, \beta\}$,

$$
\begin{aligned}
& m p_{(\tau, u), n(j-1)+1}^{k}\left(p, q, z_{0}, \ldots, z_{k-1}\right)= \\
& \quad m p_{\tau, n(j-1)+1}^{\{1, j, 2 j-1, \ldots,(n-1) j-(n-2)\}}(p, q) m p_{u, n(j-1)+1}^{k,\{1, j, 2 j-1, \ldots,(n-1) j-(n-2)\}}\left(z_{0}, \ldots, z_{k}\right)
\end{aligned}
$$

and

$$
\begin{aligned}
& e m p_{(\tau, u), n(j-1)+1}^{k}\left(p, q, z_{0}, \ldots, z_{k-1}\right)= \\
& \quad m p_{\tau, n(j-1)+1}^{\{1, j, 2 j-1, \ldots,(n-1) j-(n-2)\}}(p, q) e m p_{u, n(j-1)+1}^{k,\{1, j, 2 j-1, \ldots,(n-1) j-(n-2)\}}\left(z_{0}, \ldots, z_{k}\right),
\end{aligned}
$$

we have the following extension of Theorem 12 .

Theorem 14. Suppose $\alpha=\alpha_{1} \ldots \alpha_{j}$ and $\beta=\beta_{1} \ldots \beta_{j}$ are permutations in $S_{j}$ and $\alpha_{1}=\beta_{1}$ and $\alpha_{j}=\beta_{j}$. If $u \in\{0,1, \ldots, k-1\}^{j}$ is such that $\operatorname{red}(u)=u$ and $(\alpha, u)$ and $(\beta, u)$ have the $C_{k}$ \ $S_{n}$-minimal overlapping property, then

$$
\sum_{n \geq 0} \frac{t^{n}}{n !} \sum_{(\sigma, w) \in C_{k} \imath S_{n}} x^{(\alpha, u)-m c h((\sigma, w))} z(w)=\sum_{n \geq 0} \frac{t^{n}}{n !} \sum_{(\sigma, w) \in C_{k} 2 S_{n}} x^{(\beta, u)-m c h((\sigma, w))} z(w) .
$$

If, in addition, $p^{\operatorname{coinv}(\alpha)} q^{\operatorname{inv}(\alpha)}=p^{\operatorname{coinv}(\beta)} q^{\operatorname{inv}(\beta)}$, then

$$
\begin{gathered}
\sum_{n \geq 0} \frac{t^{n}}{[n]_{p, q} !} \sum_{(\sigma, w) \in C_{k} l S_{n}} x^{(\alpha, u)-m \operatorname{ch}((\sigma, w)} p^{\operatorname{coinv}(\sigma)} q^{\operatorname{inv}(\sigma)} z(w)= \\
\sum_{n \geq 0} \frac{t^{n}}{[n]_{p, q} !} \sum_{(\sigma, w) \in C_{k} l S_{n}} x^{(\beta, u)-m \operatorname{ch}((\sigma, w)} p^{\operatorname{coinv}(\sigma)} q^{\operatorname{inv}(\sigma)} z(w) .
\end{gathered}
$$

If $u \in\{0,1, \ldots, k-1\}^{j}$ is such that $(\alpha, u)$ and $(\beta, u)$ have the $C_{k} 2 S_{n}$-exact match minimal overlapping property, then

$$
\sum_{n \geq 0} \frac{t^{n}}{n !} \sum_{(\sigma, w) \in C_{k} l S_{n}} x^{E(\alpha, u)-m \operatorname{ch}((\sigma, w))} z(w)=\sum_{n \geq 0} \frac{t^{n}}{n !} \sum_{(\sigma, w) \in C_{k} l S_{n}} x^{E(\beta, u)-m \operatorname{ch}((\sigma, w))} z(w) .
$$

If, in addition, $p^{\operatorname{coinv}(\alpha)} q^{\operatorname{inv}(\alpha)}=p^{\operatorname{coinv}(\beta)} q^{\operatorname{inv}(\beta)}$, then

$$
\begin{gathered}
\sum_{n \geq 0} \frac{t^{n}}{[n]_{p, q} !} \sum_{(\sigma, w) \in C_{k} l S_{n}} x^{E(\alpha, u)-m c h((\sigma, w))} p^{\operatorname{coinv}(\sigma)} q^{\operatorname{inv}(\sigma)} z(w)= \\
\sum_{n \geq 0} \frac{t^{n}}{[n]_{p, q} !} \sum_{(\sigma, w) \in C_{k}\left\langle S_{n}\right.} x^{E(\beta, u)-m c h((\sigma, w))} p^{\operatorname{coinv}(\sigma)} q^{\operatorname{inv}(\sigma)} z(w) .
\end{gathered}
$$




\section{Extensions}

In this section, we shall briefly remark about two simple ways to extend the results of the previous sections. First, we can replace single occurrences of words, permutations, and $k$-colored permutations by sets of words, permutations, and $k$-colored permutations. That is, suppose $\Upsilon$ is a set of words in $\{0,1, \ldots, k-1\}^{j}$ such that $\operatorname{red}(u)=u$ for all $u \in \Upsilon$, then we say $w=w_{1} \ldots w_{n} \in\{0,1, \ldots, k-1\}^{n}$ has an $\Upsilon$-match starting at position $i$ if $\operatorname{red}\left(w_{i} \ldots w_{i+j-1}\right) \in \Upsilon$. Let $\Upsilon$-mch $(w)$ denote the number of $\Upsilon$-matches in $w$. We say that $\Upsilon$ has the $k$-minimal overlapping property if the smallest $i$ such that there exists a $w \in\{0,1, \ldots, k-1\}^{i}$ with $\Upsilon-\operatorname{mch}(w)=2$ is $2 j-1$. Similarly, if $\Upsilon$ is a set of words in $\{0,1, \ldots, k-1\}^{j}$, then we say $w=w_{1} \ldots w_{n} \in\{0,1, \ldots, k-1\}^{n}$ has an exact $\Upsilon$-match starting at position $i$ if $w_{i} \ldots w_{i+j-1} \in \Upsilon$. Let $E \Upsilon-\operatorname{mch}(w)$ denote the number of exact $\Upsilon$-matches in $w$. We say that $u$ has the $k$-exact match minimal overlapping property if the smallest $i$ such that there exists a $w \in\{0,1, \ldots, k-1\}^{i}$ with $E \Upsilon$ - $\operatorname{mch}(w)=2$ is $2 j-1$. If $\Upsilon$ is a set of permutations in $S_{j}$, then we say that a permutation $\sigma=\sigma_{1} \ldots \sigma_{n} \in S_{n}$ has an $\Upsilon$-match starting at position $i$ if $\operatorname{pred}\left(\sigma_{i} \ldots \sigma_{i+j-1}\right) \in \Upsilon$. Let $\Upsilon$-mch $(\sigma)$ denote the number of $\Upsilon$-matches in $\sigma$. We say that $\Upsilon$ has the minimal overlapping property if the smallest $i$ such that there exists a $\sigma \in S_{i}$ with $\Upsilon-\operatorname{mch}(\sigma)=2$ is $2 j-1$. If $\Upsilon$ is a set of colored permutations in $C_{k} \prec S_{n}$ such that $\operatorname{red}(u)=u$ for all $(\tau, u) \in \Upsilon$, then we say $(\sigma, w) \in C_{k} \imath S_{n}$ where $\sigma=\sigma_{1} \ldots \sigma_{n}$ and $w=w_{1} \ldots w_{n}$ has an $\Upsilon$-match starting at position $i$ if $\left(\operatorname{pred}\left(\sigma_{i} \ldots \sigma_{i+j-1}\right), \operatorname{red}\left(w_{i} \ldots w_{i+j-1}\right)\right) \in \Upsilon$ Let $\Upsilon-\operatorname{mch}((\sigma, w))$ denote the number of $\Upsilon$-matches in $(\sigma, w)$. We say that $\Upsilon$ has the $C_{k}<S_{n}$-minimal overlapping property if the smallest $i$ such that there exists a $(\sigma, w) \in C_{k} \curlyvee S_{i}$ with $\Upsilon$ - $\operatorname{mch}((\sigma, w))=2$ is $2 j-1$. If $\Upsilon$ is a set of colored permutations in $C_{k} 2 S_{n}$, then we say that $(\sigma, w) \in C_{k} 2 S_{n}$ where $\sigma=\sigma_{1} \ldots \sigma_{n}$ and $w=w_{1} \ldots w_{n}$ has an exact $\Upsilon$-match starting at position $i$ if $\left(\operatorname{pred}\left(\sigma_{i} \ldots \sigma_{i+j-1}\right), w_{i} \ldots w_{i+j-1}\right) \in \Upsilon$. Let $E \Upsilon-\operatorname{mch}((\sigma, w))$ denote the number of exact $\Upsilon$-matches in $(\sigma, w)$. We say that $\Upsilon$ has the $C_{k} \zeta S_{n}$-exact match minimal overlapping property if the smallest $i$ such that there exists a $(\sigma, w) \in C_{k} 2 S_{i}$ with $E \Upsilon$ - $\operatorname{mch}((\sigma, w))=2$ is $2 j-1$.

It should be clear that the obvious analogues of Theorem 5 in Section 3 for words $u \in\{0,1, \ldots, k-1\}^{j}$ which have the $k$-minimal overlapping or the $k$-exact match minimal overlapping property also holds for sets of words $\Upsilon \subseteq\{0,1, \ldots, k-1\}^{j}$ which have the $k$-minimal overlapping or the $k$-exact match minimal overlapping property with the same proofs. Similarly, the obvious analogues of Theorem 6 in Section 3 for $k$-colored permutations $(\tau, u) \in C_{k} 2 S_{j}$ which have the $k$-minimal overlapping or the $k$-exact match minimal overlapping property also hold for sets of $k$-colored permutations $\Upsilon \subseteq C_{k} \prec S_{j}$ which have the $k$-minimal overlapping or the $k$-exact match minimal overlapping property with the same proofs.

We also note that the proofs of Theorems 5 and 6 did not depend on the fact that we used a finite alphabet $\{0,1, \ldots, k-1\}$. That is, if $\mathbb{N}=\{0,1, \ldots\}$ is the set of natural numbers, then we can extend our definitions to words or sets of words in $\mathbb{N}^{*}$ and $\mathbb{N}$-colored permutations or sets of $\mathbb{N}$-colored permutations in the obvious way. That is, if $\Upsilon \subseteq \mathbb{N}^{j}$ has the $\mathbb{N}$-minimal overlapping property (the $\mathbb{N}$-exact match minimal overlapping property), 
then we let $\mathcal{M} \mathcal{P}_{\Upsilon, n(j-1)+1}^{\mathbb{N}}\left(\mathcal{E} \mathcal{M P}_{\Upsilon, n(j-1)+1}^{\mathbb{N}}\right)$ denote the set of all $w \in \mathbb{N}^{n(j-1)+1}$ which are maximum packings (exact match maximum packings) for $\Upsilon$. We let $C_{\mathbb{N}} 2 S_{n}$ denote the set of all pairs $(\sigma, w)$ such $\sigma \in S_{n}$ and $w \in \mathbb{N}^{n}$ and we call the elements of $C_{\mathbb{N}} 2 S_{n}$ $\mathbb{N}$-colored permutations. Then if $\Upsilon \subseteq C_{\mathbb{N}} 2 S_{n}$ has the $C_{\mathbb{N}} 2 S_{n}$-minimal overlapping property (the $C_{\mathbb{N}} 2 S_{n}$-exact match minimal overlapping property), then we let $\mathcal{M} \mathcal{P}_{\Upsilon, n(j-1)+1}^{\mathbb{N}}$ $\left(\mathcal{E} \mathcal{M P}_{\Upsilon, n(j-1)+1}^{\mathbb{N}}\right)$ denote the set of all $(\sigma, w) \in C_{\mathbb{N}} 2 S_{n}$ which are maximum packings (exact match maximum packings) for $\Upsilon$. Then we define

$$
\begin{aligned}
m p_{\Upsilon, n(j-1)+1}^{\mathbb{N}}\left(z_{0}, z_{1}, \ldots\right) & =\sum_{w \in \mathcal{M} \mathcal{P}_{\Upsilon, n(j-1)+1}^{\mathbb{N}}} z(w), \\
e m p_{\Upsilon, n(j-1)+1}^{\mathbb{N}}\left(z_{0}, z_{1}, \ldots\right) & =\sum_{w \in \mathcal{E} \mathcal{M} \mathcal{P}_{\Upsilon, n(j-1)+1}^{\mathbb{N}}} z(w), \\
m p_{\Upsilon, n(j-1)+1}^{\mathbb{N}}\left(p, q, z_{0}, z_{1}, \ldots\right) & =\sum_{(\sigma, w) \in \mathcal{M} \mathcal{P}_{\Upsilon, n(j-1)+1}^{\mathbb{N}}} p^{\operatorname{coinv}(\sigma)} q^{\operatorname{inv}(\sigma)} z(w) \text { and } \\
e m p_{\Upsilon, n(j-1)+1}^{\mathbb{N}}\left(p, q, z_{0}, z_{1}, \ldots\right) & =\sum_{(\sigma, w) \in \mathcal{E} \mathcal{M} \mathcal{P}_{\Upsilon, n(j-1)+1}^{\mathbb{N}}}^{\operatorname{coinv}(\sigma)} q^{\operatorname{inv}(\sigma)} z(w) .
\end{aligned}
$$

Then if $j \geq 3$, the following hold.

$(I)^{\mathbb{N}}$ If $\Upsilon \subseteq \mathbb{N}^{j}$ has the $\mathbb{N}$-minimal overlapping property and is such that for all $u \in \Upsilon$, $\operatorname{red}(u)=u$, then

$$
\begin{aligned}
\sum_{n \geq 0} t^{n} \sum_{w \in \mathbb{N}^{n}} x^{\Upsilon-\operatorname{mch}(w)} z(w)= & 1 \\
& \frac{1}{1-\left(\left(\sum_{i \geq 0} z_{i}\right) t+\sum_{n \geq 1} t^{n(j-1)+1}(x-1)^{n} m p_{\Upsilon, n(j-1)+1}^{\mathbb{N}}\left(z_{0}, z_{1}, \ldots\right)\right)} .
\end{aligned}
$$

$(I I)^{\mathbb{N}}$ If $\Upsilon \subseteq \mathbb{N}^{j}$ has the $k$-exact match minimal overlapping property, then

$$
\begin{aligned}
\sum_{n \geq 0} t^{n} \sum_{w \in \mathbb{N}^{n}} x^{E \Upsilon-\operatorname{mch}(w)} z(w) & = \\
& \frac{1}{1-\left(\left(\sum_{i \geq 0} z_{i}\right) t+\sum_{n \geq 1} t^{n(j-1)+1}(x-1)^{n} e m p_{u, n(j-1)+1}^{\mathbb{N}}\left(z_{0}, z_{1}, \ldots\right)\right)} .
\end{aligned}
$$

$(I I I)^{\mathbb{N}}$ If $\Upsilon \subseteq C_{\mathbb{N}} 2 S_{j}$ has the $C_{\mathbb{N}} 2 S_{n}$-minimal overlapping property and is such that for all $(\tau, u) \in \Upsilon, \operatorname{red}(u)=u$, then

$$
\begin{aligned}
\sum_{n \geq 0} \frac{t^{n}}{n !} & \sum_{(\sigma, w) \in C_{\mathbb{N}} S_{n}} x^{\Upsilon-\operatorname{mch}((\sigma, w))} p^{\operatorname{coinv}(\sigma)} q^{\operatorname{inv}(\sigma)} z(w)= \\
& \frac{1}{1-\left(\left(\sum_{i \geq 0} z_{i}\right) t+\sum_{n \geq 1} \frac{t^{n(j-1)+1}}{[n(j-1)+1]_{p, q} !}(x-1)^{n} m p_{\Upsilon, n(j-1)+1}^{\mathbb{N}}\left(p, q, z_{0}, z_{1}, \ldots\right)\right)} .
\end{aligned}
$$


$(I V)^{\mathbb{N}}$ If $\Upsilon \subseteq C_{\mathbb{N}} 2 S_{j}$ has the $C_{\mathbb{N}} 2 S_{n}$-exact match minimal overlapping property, then

$$
\begin{aligned}
\sum_{n \geq 0} \frac{t^{n}}{n !} & \sum_{(\sigma, w) \in C_{\mathbb{N}} S_{n}} x^{E \Upsilon-\operatorname{mch}((\sigma, u))} p^{\operatorname{coinv}(\sigma)} q^{\operatorname{inv}(\sigma)} z(w)= \\
& \frac{1}{1-\left(\left(\sum_{i \geq 0} z_{i}\right) t+\sum_{n \geq 1} \frac{t^{n(j-1)+1}}{[n(j-1)+1]_{q} !}(x-1)^{n} e m p_{\Upsilon, n(j-1)+1}^{\mathbb{N}}\left(p, q, z_{0}, z_{1}, \ldots\right)\right)} .
\end{aligned}
$$

\section{References}

[1] D. Beck, J. Remmel, and T. Whitehead, The combinatorics of transition matrices between the bases of the symmetric functions and the $B_{n}$ analogues, Discrete Math. 153 (1996), 3-27.

[2] F. Brenti, Permutation enumeration, symmetric functions, and unimodality, Pacific J. Math. 157 (1993), 1-28.

[3] F. Brenti, A class of $q$-symmetric functions arising from plethysm, J. Comb. Th. Ser. A, 91 (2000), 137-170.

[4] V. Dotsenko and A. Khoroshkin, Anick-type resolutions and consecutive pattern avoidance, arXiv:1002.2761v1 (2010).

[5] Ö. Eg̃eciog̃lu and J. Remmel, Brick tabloids and connection matrices between bases of symmetric functions, Discrete Applied Math. 34 (1991), 107-120.

[6] E. Egge, Restricted Colored Permutations and Chebyshev Polynomials, Discrete Math. 307 (2007), 1792-1800.

[7] S. Elizalde, Consecutive patterns and statistics on restricted permutations, Ph.D. thesis, Universitat Politécnica de Catalunya, (2004).

[8] Sergi Elizalde and Marc Noy, Consecutive patterns in permutations, Adv. in Appl. Math. 30 (2003), no. 1-2, 110-125, Formal power series and algebraic combinatorics (Scottsdale, AZ, 2001).

[9] I.P. Goulden and D.M. Jackson, Combinatorial Enumeration, A Wiley-Interscience Series in Discrete Mathematics, John Wiley \& Sons Inc, New York, (1983).

[10] Sergey Kitaev, Generalized patterns in words and permutations, Ph.D. thesis, Chalmers University of Technology and Göteborg University, 2003.

[11] Sergey Kitaev, Partially ordered generalized patterns, Discrete Math., 298 (2005), 212-229.

[12] S. Kitaev and T. Mansour, Partially ordered generalized patterns and $k$-ary words, Annals of Comb., 7 (2003), 89-100.

[13] S. Kitaev, A. Niedermaier, J.B. Remmel, and A. Riehl, New pattern matching conditions for wreath products of the cyclic groups with symmetric groups, preprint.

[14] T.M. Langley, Alternative transition matrices for Brenti's q-symmetric functions and a class of $q, t$-symmetric functions on the hyperoctahedral group, Proceedings of the 
2002 Conference on Formal Power Series and Algebraic Combinatorics, Melbourne Australia.

[15] T.M. Langley and J.B. Remmel, Enumeration of $m$-tuples of permuations and a new class of power bases for the space of symmetric functions, Advances in App. Math. 36 (2006), 30-66.

[16] T. Mansour, Pattern avoidance in coloured permutations, Sém. Lothar. Comb., 46 Article B46g, (2001).

[17] T. Mansour, Coloured permutations containing and avoiding certain patterns, Annals of Comb. $7(3)$ (2003), 349-355.

[18] T. Mansour and J. West, Avoiding 2-letter signed patterns, Sém. Lothar. Comb., 49: Article B49a, (2002).

[19] A. Mendes and J.B. Remmel, Permutations and words counted by consecutive patterns, Advances Appl. Math., 37 4, (2006), 443-480.

[20] A. Mendes and J. Remmel, Generating functions for statistics on $C_{k} 2 S_{n}$, Sém. Lothar. Comb. 54A: Article B54At, (2005/07).

[21] A. Mendes and J.B. Remmel, Generating Functions from Symmetric Functions, preprint.

[22] A. Mendes, J.B. Remmel, A. Riehl, Permutations with $k$-regular descent patterns, Permutation Patterns, (S. Linton, N. Ruškuc, V. Vatter, eds.), London. Math. Soc. Lecture Notes 376, (2010), 259-286.

[23] D. Ram, J.B. Remmel, and T. Whitehead, Combinatorics of the $q$-basis of symmetric functions, J. Comb. Th. Series A, 76(2) (1996), 231-271.

[24] R. Simion, Combinatorial statistics on type-B analogues of noncrossing partitions and restricted permutations, Electronic J. of Comb. 7(1), (2000), \#R9.

[25] J. D. Wagner, The permutation enumeration of wreath products and cyclic and symmetric groups, Advances in Appl. Math., 30 (2003), 343-368. 